\title{
A Model of Multiple Zonal Jets in the Oceans: Dynamical and Kinematical Analysis
}

\author{
P. BERLOFF*,+ \\ Department of Applied Mathematics and Theoretical Physics, University of Cambridge, Cambridge, United Kingdom, \\ and Physical Oceanography Department, Woods Hole Oceanographic Institution, Woods Hole, Massachusetts

\section{KAMENKOVICH} \\ Rosenstiel School of Marine and Atmospheric Science, University of Miami, Miami, Florida \\ J. PEDLOSKY \\ Physical Oceanography Department, Woods Hole Oceanographic Institution, Woods Hole, Massachusetts
}

(Manuscript received 9 July 2008, in final form 5 April 2009)

\begin{abstract}
Multiple alternating zonal jets observed in the ocean are studied with an idealized quasigeostrophic zonalchannel model, with the supercritical, zonal background flow imposed. Both eastward and westward background flows with vertical shear are considered. The underlying nonlinear dynamics is illuminated with analysis of the vertical-mode interactions and time-mean eddy fluxes.

Interactions between the vertical modes are systematically studied. The barotropic component of the jets is maintained by both barotropic-barotropic and baroclinic-baroclinic time-mean interactions; thus, the barotropic component of the jets cannot be accurately simulated with a randomly forced barotropic model. The roles of the vertical-mode interactions in driving the baroclinic component of the jets are also characterized. Not only the first but also the second baroclinic mode is found to be important for maintaining the baroclinic component of the jets, whereas the barotropic component of the jets is maintained mostly by the barotropic and first baroclinic modes.

The properties of the eddy forcing were systematically studied. It is shown that the baroclinic component of the jets is maintained by Reynolds stress forcing and resisted by form stress forcing only in the eastward background flow. In the westward background flow, the jets are maintained by form stress forcing and resisted by Reynolds stress forcing.

The meridional scaling and kinematical properties of the jets are studied as well as the roles of meridional boundaries. The Rhines scaling for meridional spacing of the jets is not generally confirmed, and it is also shown that there are multiple stable equilibria with different numbers of the time-mean jets. It is also found that the jets are associated with alternating weak barriers to the meridional material transport, but the locations of these barriers are not unique and depend on the direction of the background flow and depth. Finally, if the channel is closed with meridional walls, then the jets become more latent but the eddy forcing properties do not change qualitatively.
\end{abstract}

* Additional affiliation: Department of Mathematics and Grantham Institute for Climate Change, Imperial College London, London, United Kingdom.

+ Current affiliation: Department of Mathematics and Grantham Institute for Climate Change, Imperial College London, London, United Kingdom.

Corresponding author address: Pavel Berloff, Woods Hole Oceanographic Institution, Clark Laboratory, MS\#29, Woods Hole, MA 02543.

E-mail: pberloff@whoi.edu

\section{Introduction}

The principal phenomenon studied in this paper is the existence of multiple, alternating zonal jets in the oceans. The observational evidence of these jets emerged mostly over the last few years, and their theoretical understanding is incomplete. In this introduction we pose the problem, discuss the background, and describe the ocean model. The phenomenology of the modeled jets is described in section 2 . Section 3 focuses on the kinematical analysis, section 4 on the meridional scaling, and section 5 on the dynamical analysis eddies. The role of the meridional 
boundaries is addressed in section 6 , and the conclusions and discussion follow in section 7 .

\section{a. Background}

Our principal hypothesis is that the oceanic multiple jets are driven by the intrinsic nonlinear dynamics associated with mesoscale geostrophic eddies, rather than by inhomogeneities of the oceanic boundary conditions, such as wind stress pattern (Treguier et al. 2003) and topography (Nakano and Hasumi 2005). We focus on an idealized model of the jets embedded in a zonal, uniform background flow with vertical shear. The study is motivated by the existence of multiple zonal jets in oceanic gyres and in the Antarctic Circumpolar Current. We analyze the flow patterns, eddy fluxes, and material transport properties. The main distinctions of our work from the previous studies are in the more detailed analysis of the baroclinic eddy effects and the interactions between the barotropic and baroclinic vertical modes. We explore the formation of the multiple jets not only in the eastward background (EB) flow but also in the westward background (WB) flow, whereas the previous studies, motivated by the atmospheric jets, focused only on the eastward background flow. A theoretical explanation of our findings is presented in Berloff et al. (2009), where the formation, nonlinear dynamics, and equilibration of the jets are explained in terms of linear stability arguments and nonlinear self-interactions of the linear eigenmodes. Finally, we test the applicability of several important theoretical ideas, such as the potential vorticity (PV) "staircase" and the Rhines scaling for meridional structure of the jets.

The time-mean oceanic multiple zonal jets are latent jets because they are weaker than the surrounding mesoscale eddies. Because of their latency, the jets can be interpreted as preferred pathways for the zonally propagating eddies. It is plausible that the oceanic jets are dynamically similar to the multiple zonal jets observed in the atmospheres of giant gas planets, such as Jupiter (e.g., Kondratyev and Hunt 1982). However, the timemean atmospheric jets are manifest jets because they are substantially stronger than the surrounding eddies.

The observational evidence of the oceanic jets has a short history because the latent jets are difficult to observe. The zonal anisotropy of the Lagrangian float dispersion has been measured and reported over the last two decades (e.g., Krauss and Boning 1987); however, until recently these measurements failed to reveal the spatial structure of the corresponding anisotropic flow patterns (Hogg and Owens 1999; Ollitrault et al. 2006; Herbei et al. 2008). Over the last few years, the analysis of the satellite altimetry observations dramatically changed our knowledge of the multiple zonal jets (Maximenko et al.
2005; Sokolov and Rintoul 2007a; Huang et al. 2007; Schlax and Chelton 2008; Ivanov et al. 2009). Now, it is argued that the jets populate all oceans and have the time-mean velocities of a few centimeters per second. In the midlatitudes, the jets are characterized by the meridional width of about $200 \mathrm{~km}$ and by a zonal extent comparable with that of the basin. The observations also detect noticeable deviations of the jets from strict zonality (Maximenko et al. 2008; Sokolov and Rintoul 2007a). However, the vertical structure as well as the seasonal and interannual variabilities of the jets are not yet quantified.

Noneddy-resolving oceanic general circulation models (GCMs) do not simulate multiple zonal jets. The first eddy-resolving simulation that yielded some jets has been reported by Cox (1987), and more recently the jets were reported in several eddy-resolving models (Sinha and Richards 1999; Nakano and Hasumi 2005; Richards et al. 2006; Huang et al. 2007; Kamenkovich et al. 2009, hereafter KBP09). The corresponding dynamical analysis not only confirms the central role of the mesoscale eddies in maintaining the jets but also provides some guidance for refining the idealized models (KBP09).

Comprehensive reviews of the relevant theoretical ideas have been written by Rhines (1994) and Dritschel and McIntyre (2008). Rhines (1975) was the first who argued that the meridional scale (aka Rhines scale) of the barotropic jets is determined by a balance between the nonlinearity and the meridional advection of the planetary vorticity. ${ }^{1}$ Since then, all works on the jets consider the nonlinearity of the flow and the $\beta$ effect (i.e., meridional gradient of the Coriolis parameter). However, the jets might be only a weakly nonlinear phenomenon (Manfroi and Young 1999, 2002). Emergence of the jets may also depend on the first Rossby deformation radius and on the intensity and other properties of the energy cascade (Smith 2004; Theiss 2004).

In many barotropic studies, starting with Williams (1978), the jets emerge in forced-dissipative regimes driven by a spatially homogeneous, small-scale random forcing. In these studies, the central assumption is that the imposed forcing qualitatively approximates the action of the baroclinic eddies on the barotropic jets. In this type of model, the jets are formed as a result of the inverse energy cascade, and the $\beta$-effect creates strong anisotropy and channels a large fraction of the energy

\footnotetext{
${ }^{1}$ The Rhines scale is commonly thought to be the physical scale at which the inverse energy cascade in the turbulence is "arrested" by the propagating Rossby waves. On the other hand, there are arguments that most of the cascading energy overcomes the arrest and continues to cascade up to the largest scales (Huang and Robinson 1998; Sukoriansky et al. 2007).
} 
into zonal jets. Thus, the corresponding energy spectrum is strongly anisotropic (e.g., Vallis and Maltrud 1993; Chekhlov et al. 1996; Galperin et al. 2004), and the spectral nonlinear interactions are significantly nonlocal [Balk et al. (1990); see also the laboratory experiment by Wordsworth et al. (2008)]. There are flow regimes in which the meridional energy spectrum is characterized by several peaks and by large coherence of the corresponding Fourier modes (Danilov and Gurarie 2004; Danilov and Gryanik 2004). Finally, with the help of the stochastic structural stability approach, it is argued that the randomly forced barotropic jets can be described as the preferred growing structures (Farrell and Ioannou 2007, 2008).

The baroclinic components of the jets and the corresponding barotropic-baroclinic and baroclinic-baroclinic interactions are poorly understood because they require more realistic models with several vertical degrees of freedom. Panetta (1993, hereafter P93) studied multiple zonal jets in a two-layer double-periodic flow driven by a supercritical, eastward, vertically sheared background flow. He found that the jets are maintained by divergence of the momentum rather than buoyancy eddy flux ${ }^{2}$ and the meridional scaling of the jets is consistent with the Rhines scale. Also, P93 reports (i) asymmetry between the (prograde) eastward (faster but narrower) and (retrograde) westward (weaker but broader) jets ${ }^{3}$ and (ii) intrinsic low-frequency variability associated with the meridional migration, meandering, and merger of the jets. The jets were also found in a truncated P93like model (Kaspi and Flierl 2007). Finally, large-scale zonal jets were studied in wind-driven zonal-channel models with (Treguier and Panetta 1994) and without (Lee 1997) topography, but these models simulate only one or two such jets.

It has been proposed that the structure of the jets can be explained in terms of the "PV staircase" (Baldwin et al. 2007; Dritschel and McIntyre 2008; Dunkerton and Scott 2008), which stems from the inhomogeneous mixing idea of McIntyre (1982). In the staircase, the cores of the eastward jets act as partial, material transport barriers that separate broad zonal bands characterized by intense eddy-induced meridional mixing (i.e.,

\footnotetext{
${ }^{2}$ It is hard to estimate the eddy fluxes from the observations. There is evidence that some jets are maintained, whereas others are resisted by the diverging momentum fluxes (Hughes and Ash 2001).

${ }^{3}$ It is argued that in the double-periodic, barotropic decaying turbulence damped only by the high-order lateral friction, this asymmetry becomes reversed because in the westward jets, transient Rossby waves are dissipated more efficiently than in the eastward jets (Ishioka et al. 2007).
}

mixers). As a result of such inhomogeneous mixing, the meridional time-mean PV profile resembles a staircase, and the corresponding zonal velocity develops its eastwest asymmetry, ${ }^{4}$ as dictated by the PV inversion. The existence of the material transport barriers and mixers associated with strong eastward jets was confirmed in some models (Juckes and McIntyre 1987; Haynes et al. 2007; Esler 2008; Greenslade and Haynes 2008; BeronVera et al. 2008), observations (Haynes and Shuckburgh 2000; Marshall et al. 2006), and laboratory experiments (Sommeria et al. 1989). The PV profiles only resembling staircases were found in some models (Vallis and Maltrud 1993; Thompson and Young 2007; Scott and Polvani 2007) and laboratory experiments (one of the regimes reported by Read et al. 2007), but no such evidence was reported from the ocean observations and comprehensive GCMs. To what extent the oceanic jets are transport barriers and mixers is an open question.

Finding relationships between the background flow parameters and the eddy fluxes across the jets is an important issue. In some works, a scaling for the corresponding eddy diffusivity component is given in terms of the inverse energy cascade arguments, without explicit accounting for the multiple jets (Smith et al. 2002; Lapeyre and Held 2003). On the other hand, it is argued that baroclinic-barotropic interactions associated with the multiple zonal jets cause these cascade arguments to break down (Thompson and Young 2007).

The earlier mentioned modeling studies of jets were motivated by atmospheric dynamics on giant gas planets and focused on zonally unbounded flows. The effects of the meridional boundaries on properties of the jets have never been isolated and analyzed. Motivated by the oceanic rather than atmospheric jets, we will do this by solving for the jets with and without the meridional basin boundaries.

Historically, many of the idealized closed-basin studies focused on the dynamics of the wind-driven large-scale gyres, which in the upper ocean tend to mask weak zonal jets embedded in the flow. Nevertheless, these jets manifest themselves by inducing enhanced material dispersion in the zonal direction (Berloff et al. 2002). Although these jets remain poorly understood, the formation of the multiple zonal jets was studied in randomly forced, barotropic (Nadiga 2006; Kramer et al. 2006) and stratified (Berloff 2005) closed basins. In the latter study, it is argued that such jets are driven by nonlinear self-interactions of the weakly damped basin modes. Overall, the effects

\footnotetext{
${ }^{4}$ An alternative argument explaining the velocity asymmetry exploits the analogy with hydraulically controlled flow and thus relates velocity and the width of the jet (Army 1989).
} 


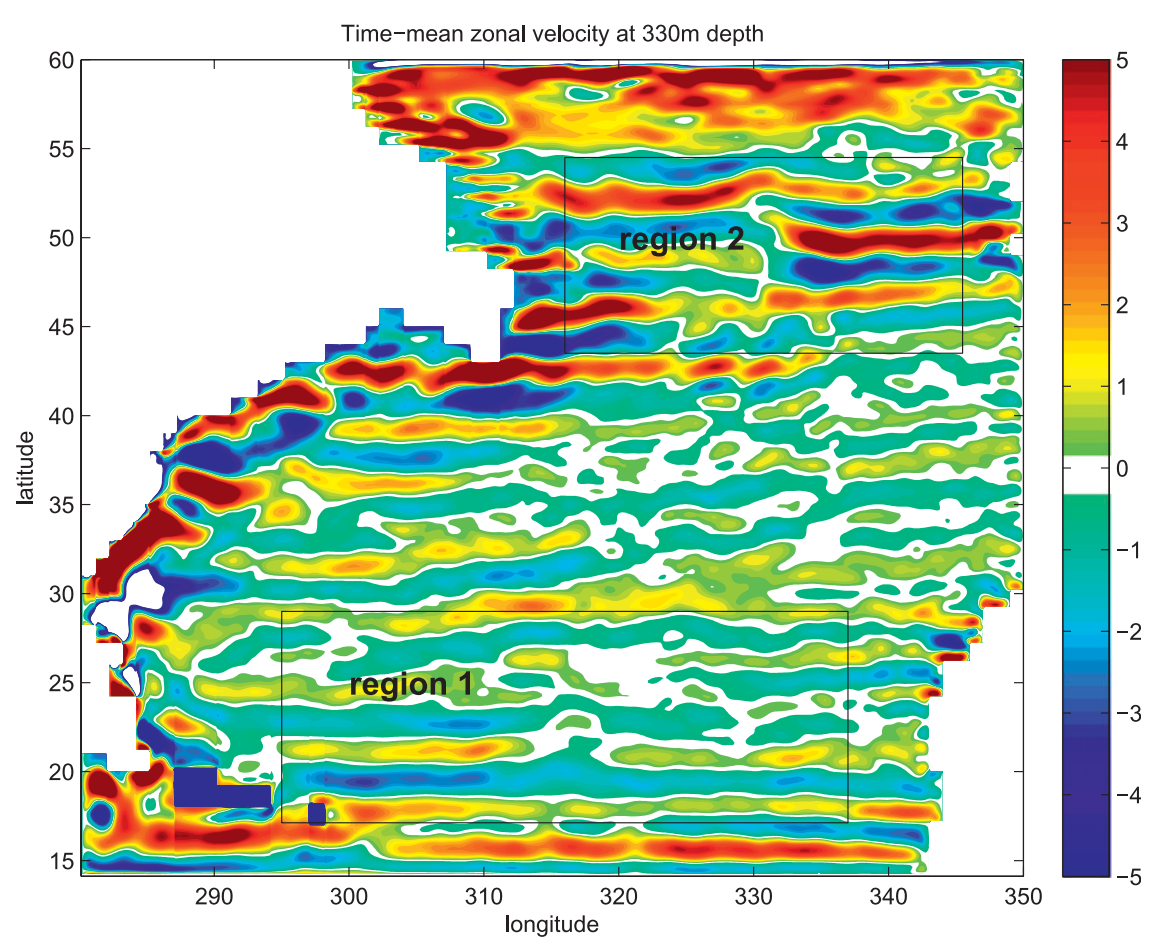

FIG. 1. Multiple zonal jets simulated by a comprehensive, eddy-resolving GCM of the North Atlantic (KBP 2009). Shown is zonal velocity, averaged over 9 yr, at 500-m depth. The schematic rectangles 1 and 2 indicate regions dominated by the westward and eastward upper-ocean background flows, respectively.

of the meridional boundaries on properties of the jets have never been isolated and analyzed, and, in this study, we will do this by solving for the jets with and without the meridional basin boundaries.

There are other types of multiple jets that are similar to the jets, which are the focus of this paper. The equatorial currents (Firing 1987) might be dynamically related to the midlatitude, multiple zonal jets through instabilities of large-scale waves (e.g., Hua et al. 2008). Multiple-jet zonation of currents is also observed on continental slopes (Franco et al. 2008), suggesting the important role for the topographic $\beta$ effect. Finally, vigorous research on zonal jets is carried out by the plasma physics community - many aspects of this effort have geophysical counterparts; a comprehensive review of the subject is in Diamond et al. (2005).

In summary, past studies have not yielded a universally accepted view on the origins and dynamics of the multiple zonal jets observed in the oceans (and atmospheres). Nearly all theories argue that the jets are a nonlinear phenomenon driven by the mesoscale eddies in the presence of the meridional gradient of the planetary vorticity, but most theories focus on the barotropic dynamics and neglect the effects of the background flow and lateral boundaries.

\section{b. Ocean model}

This study aims to analyze the multiple zonal jets embedded in zonal oceanic flows, such as those simulated in the comprehensive, eddy-resolving GCM (KBP09) and shown in Fig. 1. In these solutions, the multiple jets are more pronounced in the southern half of the subtropical gyre, where the background flow is westward, and in the region of $40^{\circ}-55^{\circ} \mathrm{N}$, where the background flow is eastward. In both of these regions, the background flow is upper-ocean intensified and nearly zonal; also, it does not change the sign with depth and decays to zero near the bottom. Our choice of the idealized model and the background flow configuration is motivated by these characteristics: we establish the simplest, but physically relevant, starting point; the more physical complexity can be systematically added later on. For most of the study, the basin is configured as a zonally periodic channel with a flat bottom; this imposes zonal homogeneity of the time-mean eddy fluxes and, thus, greatly simplifies the initial analysis. A closed-basin extension of the model is considered as well, later, for assessing the effects of the meridional boundaries. In this paper we diagnose the jet patterns, the material transport properties of the flow, and the eddy dynamics—both for the eastward and 
westward background flows and also for a broad range of model parameters. However, the underlying theory, which relies on the linear stability arguments, is presented in the sequel paper by Berloff et al. (2009).

The meridional width of the reference channel is $L_{y}=$ $1800 \mathrm{~km}$, but some solutions with $L_{y}=3600 \mathrm{~km}$ are also discussed. The channel is zonally periodic, with the period $L_{x}=2 L_{y}$. The total depth of fluid is $H=4 \mathrm{~km}$. The background planetary vorticity gradient is $\beta=$ $2 \times 10^{-11} \mathrm{~m}^{-1} \mathrm{~s}^{-1}$, and the midchannel $\left(45^{\circ} \mathrm{N}\right)$ Coriolis parameter is $f_{0}=0.83 \times 10^{-4} \mathrm{~s}^{-1}$. The bottom friction $\gamma$ is varied from zero to $4 \times 10^{-7} \mathrm{~s}^{-1}$, and its reference value is zero. The eddy viscosity $\nu$ is varied from 50 to $400 \mathrm{~m}^{2} \mathrm{~s}^{-1}$ (this range is typical of eddy-resolving GCMs), and its reference value is $100 \mathrm{~m}^{2} \mathrm{~s}^{-1}$.

The stratification is approximated with either two or three stacked isopycnal layers, thus allowing for the systematic study of the importance of the second baroclinic mode. In the two-layer case, the layer depths are $H_{1}=1$ and $H_{2}=3 \mathrm{~km}$, starting from the top; in the three-layer case, the depths are $H_{1}=1, H_{2}=1$, and $H_{3}=2 \mathrm{~km}$, respectively. The reduced gravities $g_{1}^{\prime}$ and $g_{2}^{\prime}$ are associated with the density jumps across the upper and lower interfaces between the isopycnal layers, and $g_{2}^{\prime}$ exists only in the three-layer model. The stratification parameters of the three-layer model are as follows:

$$
\begin{aligned}
& S_{1}=\frac{f_{0}^{2}}{H_{1} g_{1}^{\prime}}, \quad S_{21}=\frac{f_{0}^{2}}{H_{2} g_{1}^{\prime}}, \quad S_{22}=\frac{f_{0}^{2}}{H_{2} g_{2}^{\prime}}, \quad \text { and } \\
& S_{3}=\frac{f_{0}^{2}}{H_{3} g_{2}^{\prime}},
\end{aligned}
$$

and the reduced gravities are chosen so that the first and the second baroclinic Rossby deformation radii, $\mathrm{Rd}_{1}$ and $\mathrm{Rd}_{2}$, are 25 and $12 \mathrm{~km}$, respectively. In the two-layer model, there are only two stratification parameters, $S_{1}$ and $S_{2}=S_{21}$, and $g_{1}^{\prime}$ is chosen so that the only deformation radius $\mathrm{Rd}_{1}=g_{1}^{\prime} \sqrt{H_{1} H_{2}} / f_{0} \sqrt{H_{1}+H_{2}}$ is $25 \mathrm{~km}$.

The quasigeostrophic equations (Pedlosky 1987) for three dynamically active isopycnal layers are as follows:

$$
\begin{aligned}
& \frac{\partial q_{1}}{\partial t}+J\left(\psi_{1}, q_{1}\right)+\beta \frac{\partial \psi_{1}}{\partial x}=\nu \nabla^{4} \psi_{1}+D_{1}^{\mathrm{LS}}, \\
& \frac{\partial q_{2}}{\partial t}+J\left(\psi_{2}, q_{2}\right)+\beta \frac{\partial \psi_{2}}{\partial x}=\nu \nabla^{4} \psi_{2}+D_{2}^{\mathrm{LS}}, \quad \text { and } \\
& \frac{\partial q_{3}}{\partial t}+J\left(\psi_{3}, q_{3}\right)+\beta \frac{\partial \psi_{3}}{\partial x}=\nu \nabla^{4} \psi_{3}+D_{3}^{\mathrm{LS}}-\gamma \nabla^{2} \psi_{3},
\end{aligned}
$$

where the layer index starts from the top, $J(\cdot)$ is the Jacobian operator, $D_{i}^{\mathrm{LS}}$ is the large-scale dissipation, and the last term in (4) is the bottom friction. The isopycnal PV anomalies $q_{i}$ are related to the velocity streamfunctions $\psi_{i}$ through the elliptic, PV inversion subproblem

$q_{1}=\nabla^{2} \psi_{1}+S_{1}\left(\psi_{2}-\psi_{1}\right)$,

$q_{2}=\nabla^{2} \psi_{2}+S_{21}\left(\psi_{1}-\psi_{2}\right)+S_{22}\left(\psi_{3}-\psi_{2}\right)$, and

$q_{3}=\nabla^{2} \psi_{3}+S_{3}\left(\psi_{2}-\psi_{3}\right)$.

The two-layer modification of the model is formulated similarly:

$$
\begin{aligned}
\frac{\partial q_{1}}{\partial t}+J\left(\psi_{1}, q_{1}\right)+\beta \frac{\partial \psi_{1}}{\partial x} & =\nu \nabla^{4} \psi_{1}+D_{1}^{\mathrm{LS}} \\
\frac{\partial q_{2}}{\partial t}+J\left(\psi_{2}, q_{2}\right)+\beta \frac{\partial \psi_{2}}{\partial x} & =\nu \nabla^{4} \psi_{2}+D_{2}^{\mathrm{LS}}-\gamma \nabla^{2} \psi_{2} \\
q_{1} & =\nabla^{2} \psi_{1}+S_{1}\left(\psi_{2}-\psi_{1}\right), \quad \text { and } \\
q_{2} & =\nabla^{2} \psi_{2}+S_{2}\left(\psi_{1}-\psi_{2}\right)
\end{aligned}
$$

The isopycnal velocity components are found from the velocity streamfunction as follows:

$$
u_{i}=-\frac{\partial \psi_{i}}{\partial y} \quad \text { and } \quad v_{i}=\frac{\partial \psi_{i}}{\partial x}
$$

The dynamical equations are solved either in their original, isopycnal-layer form or in terms of their verticalmode equivalence (McWilliams 2006). No-slip lateralboundary conditions are used for each isopycnal layer. The mass and momentum constraints are imposed following McWilliams (1977).

The forcing in the governing equations is introduced through the imposed, background velocity with vertical shear (Haidvogel and Held 1980; P93):

$$
\psi_{i} \rightarrow-U_{i} y+\psi_{i}
$$

where $U_{i}$ is the background zonal velocity. The forcing can also be interpreted as the imposed meridional density contrast across the channel. We always set the deepestlayer background velocity to zero, and in the three-layer model, we set $U_{2}=0.5 U_{1}$. Given (13), the governing equations are rewritten with respect to perturbations $\psi_{i}$ and $q_{i}$ around the background flow. The background velocity $U_{i}$ is horizontally uniform, which ensures that the characteristic length scales of the multiple jets are not imposed but, instead, are internally generated by the intrinsic flow dynamics. The background flow configuration is linearly unstable for the parameters of interest and therefore the flow solutions are full with transient eddies. 
TABLE 1. Parameters used in the two-layer channel model. The other parameters are kept fixed: $L_{x}=2 L_{y}, \mathrm{Rd}_{1}=25 \mathrm{~km}, H_{1}=1$ and $H_{2}=3 \mathrm{~km}$, and $U_{2}$ is zero.

\begin{tabular}{|c|c|c|c|c|c|c|c|}
\hline & $\mathrm{EB} U_{1}$ & $\mathrm{WB} U_{1}$ & $\beta \times 10^{11}$ & $\nu$ & $\gamma \times 10^{7}$ & $\lambda \times 10^{7}$ & $L_{y}$ \\
\hline Reference value & $+6 \mathrm{~cm} \mathrm{~s}^{-1}$ & $-3 \mathrm{~cm} \mathrm{~s}^{-1}$ & $2 \mathrm{~m}^{-1} \mathrm{~s}^{-1}$ & $100 \mathrm{~m}^{2} \mathrm{~s}^{-1}$ & $0 \mathrm{~s}^{-1}$ & $2 \mathrm{~m}^{-2} \mathrm{~s}^{-1}$ & $1800 \mathrm{~km}$ \\
\hline Variation & $+4 \div+10$ & $-1.5 \div-8$ & $0.3 \div 3$ & $60 \div 500$ & $0 \div 4$ & $0 \div 20$ & $L_{y} \rightarrow 2 L_{y}$ \\
\hline
\end{tabular}

We impose large-scale damping,

$$
D_{i}^{\mathrm{LS}}=\lambda_{i j} \psi_{j}
$$

that selectively acts on the largest scales of motion. In some previous studies of the multiple zonal jets, largescale damping is included either explicitly (e.g., Danilov and Gurarie 2004) or implicitly in the form of thermal radiative cooling (e.g., P93). In our model, $\lambda_{i j}$ is chosen so that if (14) is projected on the vertical modes, then $\lambda_{i j}$ becomes the diagonal and isotropic tensor with the magnitude given by the large-scale damping rate $\lambda$. We assessed the effect of the large-scale damping by setting $\lambda$ to zero, but the reference value of $\lambda$ is $1 \times 10^{-7} \mathrm{~m}^{-2} \mathrm{~s}^{-1}$. In the absence of bottom friction, this damping accounts for only $3 \%$ of the total energy dissipation but it prevents a very slow (on the time scale of centuries) merger of the multiple zonal jets into the broad jet in the center with two return jets around it. A similar effect can be obtained by very weak bottom friction, which accounts for only about $6 \%$ of the total energy dissipation in the model. Although we varied the bottom friction in the sensitivity study, our main focus is on the multiple-jet flow regime, which is dominated by the lateral dissipation and is the same regime studied in KBP09. At this point, $D_{\mathrm{LS}}$ crudely accounts for the large-scale energy sink associated with the missing meridional boundary layers.

We studied dependence on the main parameters, but most of the presentation focuses on the two reference solutions-for the EB and WB flows (Table 1). The EB flow is twice stronger than the WB flow, and each flow is about 1.5 times stronger than the corresponding critical flow. Significant differences between the single-jet dynamics in the EB and WB flows were first reported by Ivchenko et al. (1997) but overall the WB flows were significantly less studied in the past.

The governing equations are discretized with secondorder finite differences, and the formulation of the Jacobians is PV flux conserving. The prognostic equations are marched in time with the leapfrog scheme and 0.5 -h time step, and the elliptic PV inversion problem is solved for the corresponding velocity streamfunctions on each time step by a direct solver. The horizontal grid resolution is uniform with $7-\mathrm{km}$ resolution $(512 \times 257$ grid points for the reference solutions). The statistically equilibrated regimes are reached after $20-40 \mathrm{yr}$ of integration, and our analysis is based on the subsequent $100 \mathrm{yr}$ of integration.

Dynamical analysis of the flow solutions is based on calculating eddy fluxes of PV and its components relative vorticity

$$
R_{i}=\nabla^{2} \psi_{i}
$$

and isopycnal stretching (take $S_{22}=S_{3}=0$ in the twolayer model)

$$
\begin{aligned}
& B_{1}=S_{1}\left(\psi_{2}-\psi_{1}\right), \\
& B_{2}=S_{21}\left(\psi_{1}-\psi_{2}\right)+S_{22}\left(\psi_{3}-\psi_{2}\right), \text { and } \\
& B_{3}=S_{3}\left(\psi_{2}-\psi_{3}\right),
\end{aligned}
$$

which corresponds to the local anomaly of the buoyancy. The flow solutions are decomposed into the time-mean $\overline{\psi_{i}}$ and fluctuation $\psi_{i}^{\prime}$ components; the fluctuations are referred to as the mesoscale eddies. In the $i$ th isopycnal layer, the time-mean eddy PV flux is defined as

$$
\overline{\mathbf{f}_{i}}(x, y)=\overline{\mathbf{u}_{i}^{\prime} q_{i}^{\prime}},
$$

and the eddy fluxes of $R, \overline{\mathbf{f}_{R_{i}}}$, and $B, \overline{\mathbf{f}_{B i}}$, are defined similarly. The time-mean eddy forcing term

$$
F_{i}(x, y)=-\nabla \overline{\mathbf{f}_{i}}
$$

can be interpreted as internally generated PV forcing that maintains the multiple zonal jets. Minus divergences of $\overline{\mathbf{f}_{R}}$ and $\overline{\mathbf{f}_{B}}$ are the Reynolds stress forcing, $F_{R_{i}}$, and form stress forcing, $F_{B_{i}}$, respectively. In the channel, total eddy forcing is always balanced by the dissipation terms, which are completely dominated by the lateral friction.

\section{Main features of the multiple jets}

The flow solutions (Fig. 2) can be characterized as follows. The time-mean jets are asymmetric in the sense that the eastward jets are faster and narrower than the westward jets, but the total eastward and westward mass transports are always equal as a result of the zonal momentum conservation. We focus on the channel with $L_{y}=1800 \mathrm{~km}\left(72 \mathrm{Rd}_{1}\right)$, but some benchmark solutions are found for $L_{y}=3600 \mathrm{~km}\left(144 \mathrm{Rd}_{1}\right)$ to demonstrate that as long as there are many jets in the channel, the 
(a)
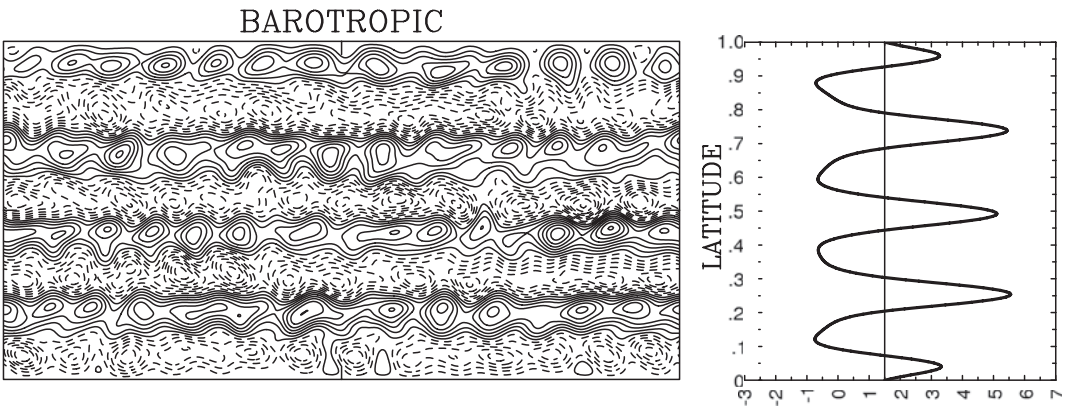

(b)
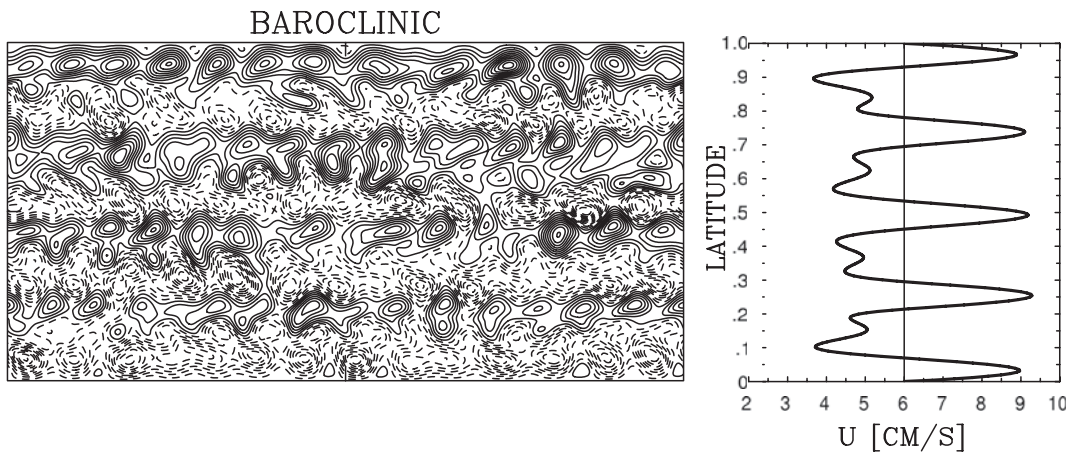

FIG. 2. Multiple-jet flow in the two-layer zonal channel. Instantaneous (a) barotropic and (b) baroclinic velocity streamfunctions of the EB flow reference solution [contour interval $(\mathrm{CI})=$ 2 Sv]. (right) The corresponding time-mean zonal velocity profiles. Straight lines indicate the background velocities. Latitude values are normalized by the width of the channel $\left(L_{y}=\right.$ $\left.1800 \mathrm{~km}=72 \mathrm{Rd}_{1}\right)$.

phenomenology and dynamics are largely insensitive to $L_{y}$ (Fig. 3). On the other hand, the solutions are moderately sensitive to the initial conditions because the model allows for the multiple equilibria characterized by different numbers of the jets in the channel (Fig. 3; section 4).

Despite some similarities, the EB and WB flow regimes are very different. In the EB flow, the barotropic and baroclinic jet components flowing in the same direction are located on the same latitudes-that is, they reinforce each other-whereas in the WB flow they oppose each other. As a result of this behavior, deep-ocean jets are weaker/stronger than the upper-ocean jets in the EB/WB flow. Finally, between the EB and WB flows, there are fundamental dynamical and kinematical differences discussed further later.

The importance of the second baroclinic mode is studied by comparing the two- and three-layer versions of the model (section 1b). The corresponding solutions have important similarities and differences (Fig. 4; compare with Fig. 2). The similarity of the meridional jet structures allows us to focus the presentation on the twolayer flow regimes and to make connections to the threelayer regimes only when necessary. The main difference is in the significant reduction of the barotropic component of the jets in the presence of the second baroclinic mode. This result undermines the common assumption (section 1a) that the structure of the barotropic component of the jets can be properly captured with a purely barotropic model (in section 5, we again demonstrate the importance of baroclinic-baroclinic interactions for the barotropic dynamics). In the WB flow, the second baroclinic mode plays a more important role than in the EB flow. This is due to the enhanced importance of the deep-ocean flow, which is better represented with the increased vertical resolution. Important dynamical roles of the second baroclinic mode are addressed in section 5 .

\section{Lagrangian analysis and PV washboard}

This section discusses the meridional structure of the time-mean PV, relates it to the meridional, eddy-induced material transport, and connects these results to the PV staircase paradigm (section 1a).

The PV staircase paradigm - commonly discussed in the context of eastward background flows-implies that the cores of the prograde (i.e., adding to the background flow) jets tend to behave as barriers to the meridional material transport, whereas the retrograde (i.e., subtracting from the background flow) jets behave as mixers (i.e., surf zones) of the meridional material transport. Because, in the absence of diabatic sources, $\mathrm{PV}$ is a 

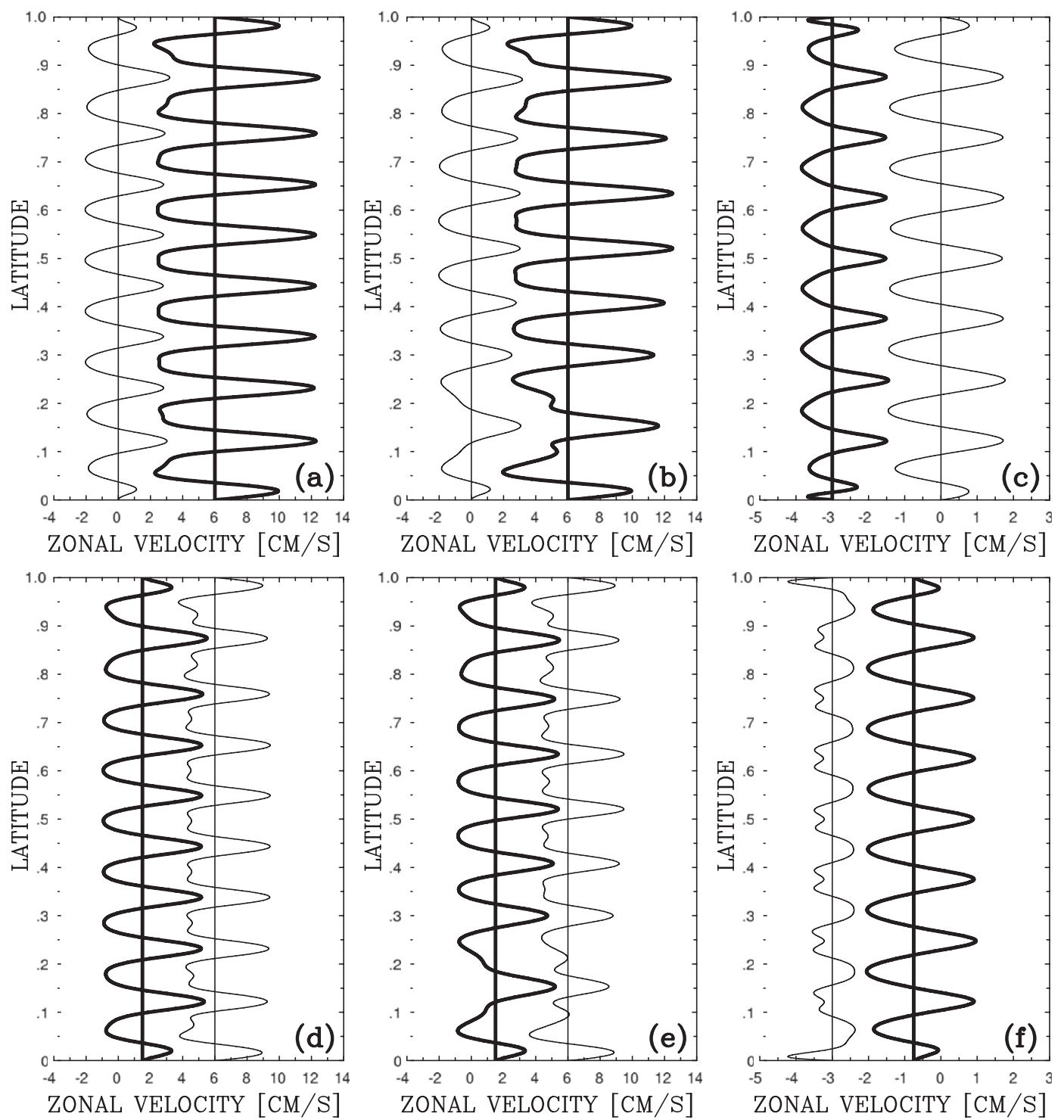

FIG. 3. Time-mean zonal velocity components in the broad channel. (top) The upper-ocean (thick line) and deepocean (thin line) velocity components. (bottom) The corresponding barotropic (thick line) and baroclinic (thin line) velocity components. Multiple EB flow solutions with either (a),(d) 10 or (b),(e) 9 eastward jets. These solutions are the broad-channel equivalents of the solution in Fig. 2. (c),(f) WB flow solution is also shown. Straight lines indicate the background flow velocities. Latitude values are normalized by the width of the channel $\left(L_{y}=3600 \mathrm{~km}=144 \operatorname{Rd}_{1}\right)$.

materially conserved quantity, these barriers and mixers, if they are very strong, should be associated with the evident meridional PV steps and interstep zones, respectively. Here, the snag is that most of the studies predicting the staircase are backed up by the single-layer models, and it remains to be shown that the staircase can be reached in more realistic models.

In the presence of a regular array of multiple zonal jets, it is not surprising to see a nearly periodic deformation of the corresponding meridional PV profile. More important is how close this profile approaches the ideal staircase, which corresponds to broad regions of homogenized, zero-gradient PV separated by steep PV steps with the infinite gradient. Within the broad range of explored parameters, we find that PV profiles do not approach the ideal staircase (Fig. 5). Instead, these profiles can be characterized as PV "washboards," which are weaker in the WB flow regime. Unlike the staircase, these washboards exhibit partially homogenized zones that separate equally broad zones with modestly enhanced PV gradient. The latter gradient, however, exceed the background PV gradient by a modest 

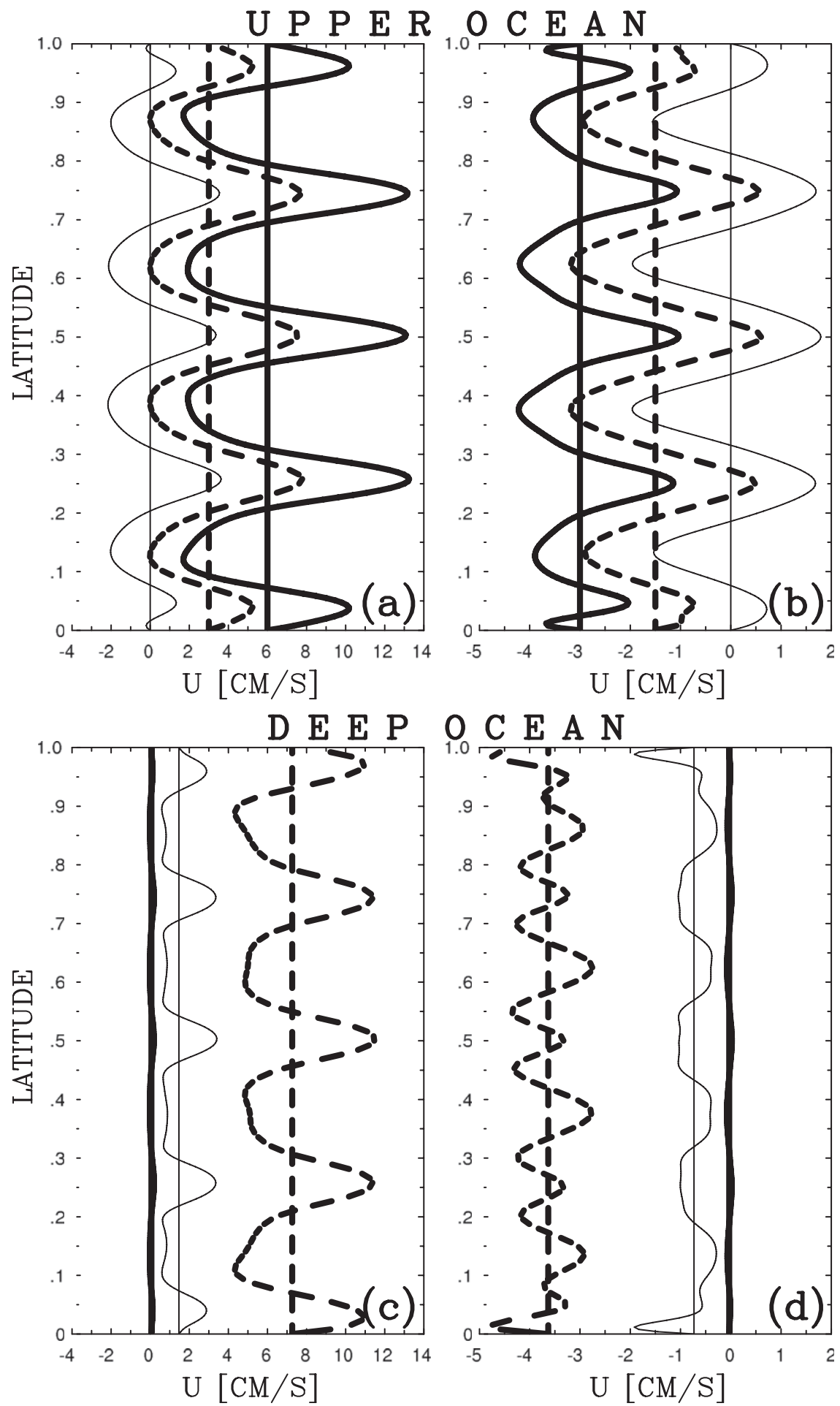

FIG. 4. Time-mean zonal velocities of the three-layer channel model. Isopycnal-layer velocities corresponding to the (a) EB and (b) WB flow reference solutions are shown in the upper-ocean (thick curve), middle-ocean (dashed thick curve), and deep-ocean layers (thin curve). (c),(d) The corresponding barotropic (thick curve), first baroclinic (dashed thick curve), and second baroclinic (thin curve) velocities. Vertical lines indicate the corresponding background flow velocities. 

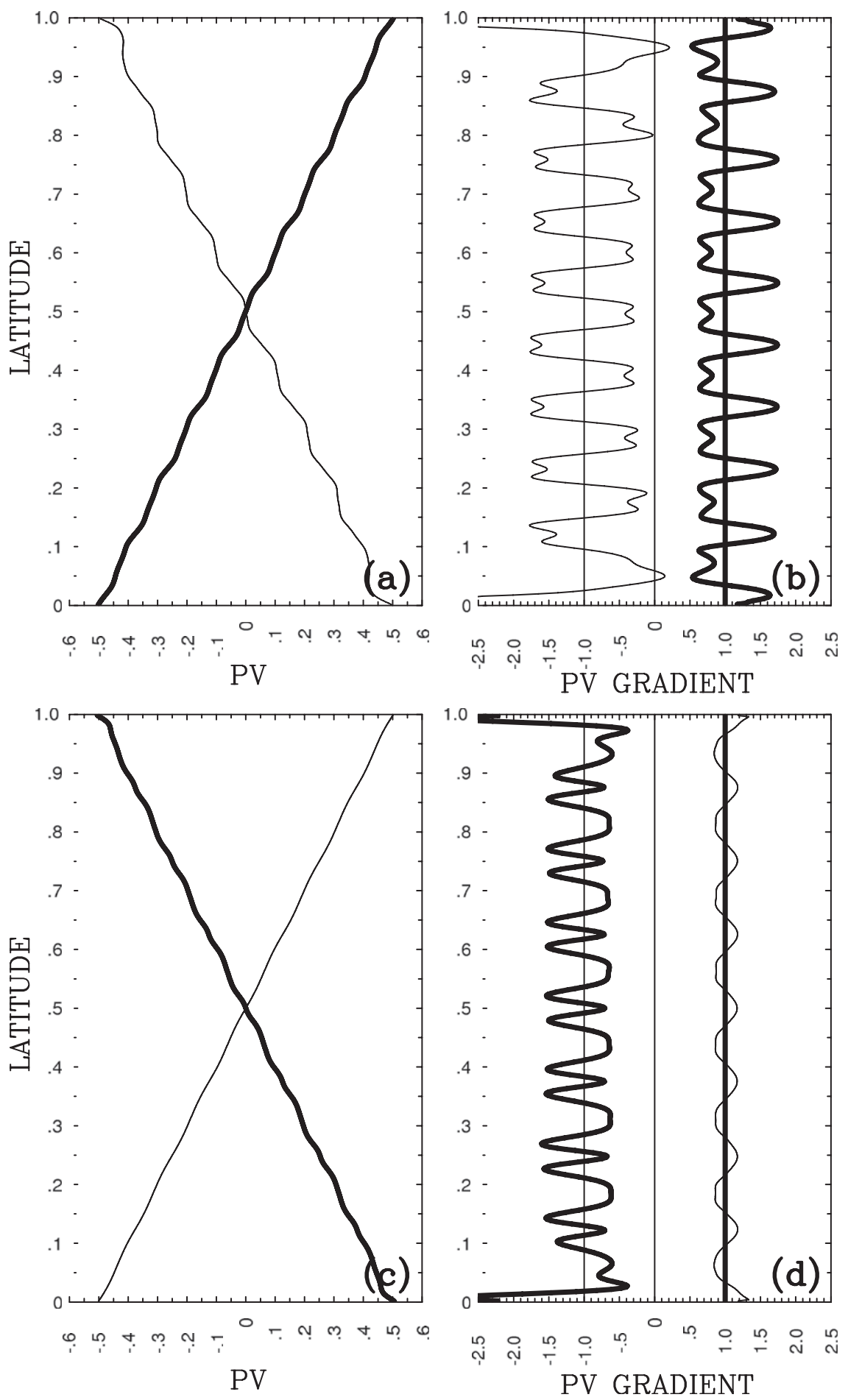

FIG. 5. Meridional structure of the time-mean PV. The panels corresponds to the EB and WB flow solutions from Figs. 3a,c. (a),(c) The upper-ocean (thick) and deep-ocean (thin curve) PV profiles; (b),(d) the corresponding PV gradients. The upper- and deep-ocean PV profiles are normalized by $L_{y}\left|\beta+S_{1} U_{1}\right|$ and $L_{y}\left|\beta-S_{2} U_{1}\right|$, respectively; hence, the corresponding background gradients (indicated by straight lines) are either +1 or -1 , depending on the sign of the background PV gradient. 
factor of 2 or smaller. A more detailed inspection reveals that some of these partial barrier and mixing zones incorporate more localized and weaker secondary barrier and mixing zones, suggesting that the staircase description is even less relevant.

To clarify the relationship between the washboard and the eddy-induced, meridional material transport, we carried out the Lagrangian analysis of the flow. The methodology is as follows: The transport of material is represented by Lagrangian particles uniformly distributed in space and advected by the flow velocity. Time integration of the particle trajectories is performed by fourth-order Runge-Kutta method, with the flow velocity obtained by the bicubic spatial interpolation of the velocity streamfunction field. For each latitude and isopycnal layer, we find an ensemble of particles that, by the end of the specified time interval, have migrated across the latitude of interest in either the northward or southward direction. Each ensemble incorporates several realizations that differ by the timing at which the particles were deployed in the evolving flow. We find that the time interval of 100 days, 500 realizations, 1000 particles in each realization, and 1-day intervals between the particle releases gives reliable statistics.

The total meridional material flux $M_{i}^{\text {tot }}(y)$ is estimated by dividing the total number of particles in the corresponding ensemble by the time interval and by the number of realizations. A component of this flux needs to be sorted out because it can be interpreted as reversible. The reversibility is associated with coherent blobs of fluid that migrate across the latitude of interest but do not cross a PV isoline that evolves in time and corresponds to the time-mean PV on this latitude. The irreversible component $M_{i}^{\mathrm{irrev}}(y)$ of the total flux is obtained by counting only those particles that eventually crossed the corresponding instantaneous PV isoline. Fluxes $M_{i}^{\text {tot }}(y)$ and $M_{i}^{\text {irrev }}(y)$ calculated from the reference solutions are shown in Fig. 6. The irreversible flux can be a small fraction of the total flux, and it is a more accurate detector of the barriers and mixers.

In the EB regime, the cores of the upper-ocean prograde (eastward) jets manifest themselves as weak transport barriers separated by the mixing zones. However, in the deep ocean the picture is the opposite: the eastward jets act as the mixing zones separated by the barriers associated with the westward jets. In the WB regime, the upper-ocean barriers-located between the prograde (westward) and retrograde (eastward) jet cores-are the weakest. The upper-ocean prograde jets and the cores of the retrograde jets behave as very weak mixers. In the deep ocean, the barrier/mixer contrast is noticeably larger; the mixing zones are located only on the prograde (relative to $U_{1}$ ) jets, and the barriers are lo- cated on the southern flanks of the prograde jets. Overall, the permeability contrast between the barriers and mixers - responsible for bending the washboard—can be characterized by factors of about 4 and 1.5 in the EB and WB regimes, respectively.

We confirmed the configurations of the barriers and mixers with a different methodology, by solving for the meridional eddy fluxes of the passive tracer concentration. The corresponding tracer equilibrium was maintained by imposing the tracer source/sink with the uniform meridional gradient. We also calculated effective diffusivities (Shuckburgh and Haynes 2003; Greenslade and Haynes 2008), and they yielded qualitatively similar results.

The existence of the weak barriers in the upper-ocean eastward jets (of the EB regime) is consistent with the observed, slightly different but distinct, bands of chlorophyll concentrations in the multiple jets of the Southern Ocean (Sokolov and Rintoul 2007b); however, more detailed observational evidence of the jets' permeability is not available, so far. The weak barriers and mixers simulated by our idealized model are consistent with those simulated by the comprehensive eddy-resolving GCM (KBP09).

To prove that our time-mean flow is indeed very far from the ideal staircase, we solved for the velocity profiles corresponding to the ideal PV staircases fitted in both isopycnal layers. The inversion of this PV into velocity was made with the elliptic solver from the model. The outcome yielded a qualitatively incorrect velocity field and more so in the WB regime. The corresponding EB velocity profiles, illustrated by Fig. 7, should be compared with the actual velocity profiles from Figs. 3a,c: on the same latitudes, deep-ocean jets emerging on the top of the background flow are in the opposite direction to the upper-ocean jets, and the amplitudes of the jets are also predicted incorrectly. This is so because the actual jets in the model have equally important barotropic and baroclinic modes, whereas the implied two-layer staircase significantly underpredicts the barotropic mode.

The inhomogeneity of the meridional material fluxes results into inhomogeneity of the eddy PV fluxes and their components (Figs. 11, 12). In all solutions, eddy PV and buoyancy fluxes are directed down gradient relative to the corresponding time-mean quantities; in this sense, these fluxes are diffusive (although with the nonuniform diffusivities). Relative-vorticity fluxes, in contrast, can be both down and up gradient. In the EB flow, $\overline{\mathbf{f}_{R}}$ and $\overline{\mathbf{f}_{B}}$ nearly cancel each other in the cores of the prograde jets, where they have well-defined local extrema. These partial eddy PV flux barriers, which correspond to this cancellation, are consistent with the associated weak 

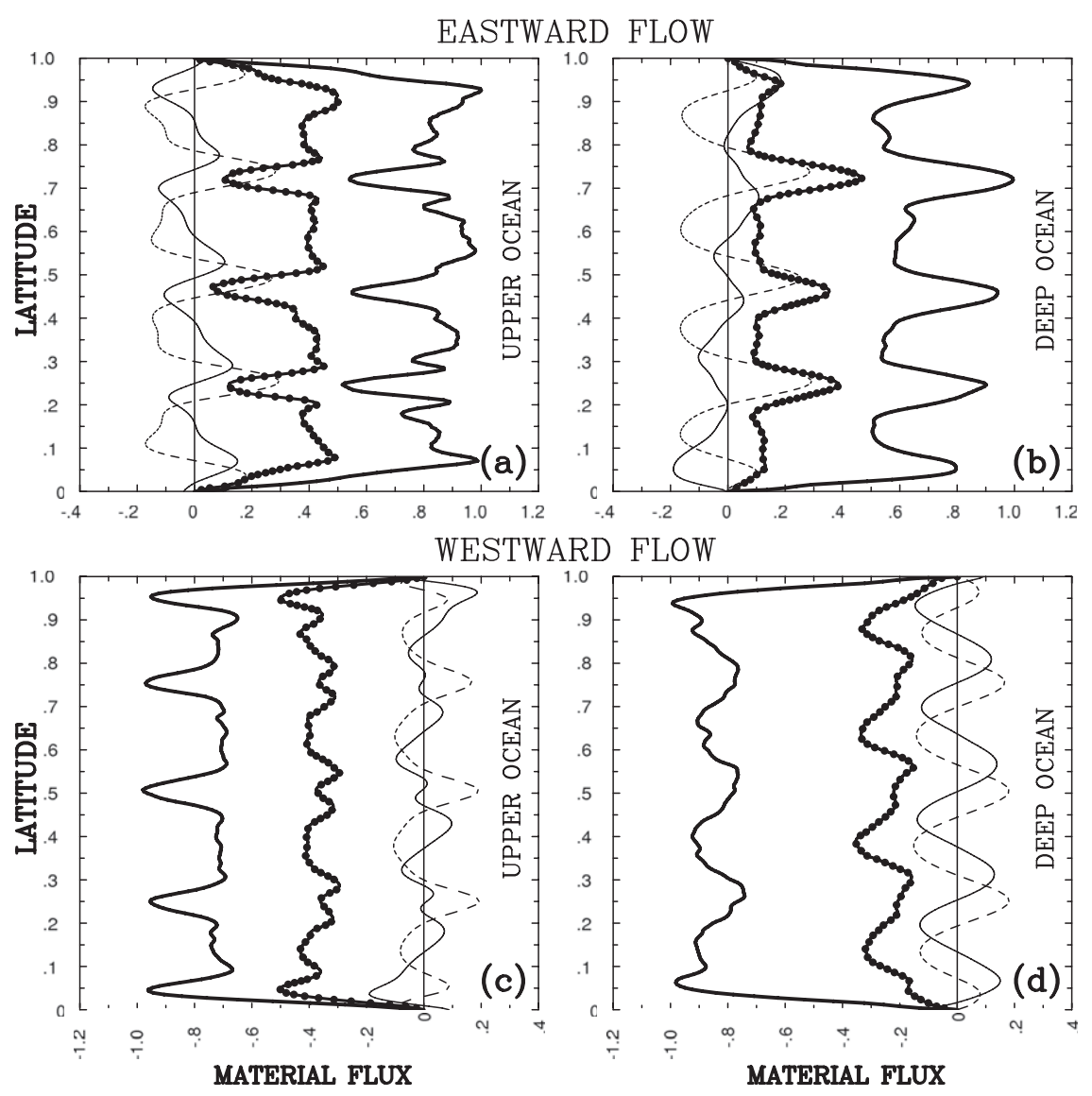

FIG. 6. Meridional material transport. Total $M_{i}^{\text {tot }}$ and irreversible $M_{i}^{\text {irrev }}(y)$ material fluxes are shown with thick curve and thick curve with filled circles, respectively. Both fluxes are normalized by the maximum value of the corresponding total flux. The corresponding profiles of the time-mean PV (thin) and zonal velocity (dashed curve) anomalies are shown for convenience, with arbitrary amplitudes, (a) upper-ocean and (b) deep-ocean material fluxes for the reference EB flow solution. (c),(d) Corresponds to the reference WB flow solution; because the background flow is negative, the corresponding material fluxes are multiplied by -1 , for convenience.

material transport barriers. In the deep ocean, the weak barriers and mixers exchange their locations, and the up gradient $\overline{\mathbf{f}_{R}}$ competes with the $\overline{\mathbf{f}_{B}}$ in the retrograde jets. In the WB flow, in terms of the eddy flux patterns, the upper layer plays the role of the EB flow deep layer (Fig. 12). The contrast between the barriers and mixers is relatively weak, and there are meridional high-frequency variations associated with $\overline{\mathbf{f}_{B}}$. All of this is consistent with the material transport analysis of this section.

To summarize, we have found that the description of the time-mean meridional PV in terms of the staircase is not accurate. The observed PV pattern-referred to as the washboard-is associated with weak and alternating material transport barriers and mixers. The locations of the barriers and mixers depend on direction of the background flow and depth, and the barriers are not necessarily located on the prograde jets. The time-mean flow does not approach the ideal PV staircase because of the strong barotropic mode predicted by the dynamics.

\section{Meridional scale of the jets}

In this section, we demonstrate that the multiple jets are a robust feature of the model. Also, we show that the empirical width of the jets does not scale with Rhines scaling, which is based on the nonlinear inverse cascade arguments. We address both issues by studying the sensitivity of the jet width to parameters of the problem.

We define the meridional jet scale $L_{j}$ as the length scale corresponding to the mean wavenumber (i.e., the one corresponding to the median value in the spectrum) $k_{j}$ of the meridional power spectrum $E(k)$ of the time-mean and zonally averaged, zonal barotropic velocity. The barotropic component of velocity is chosen 

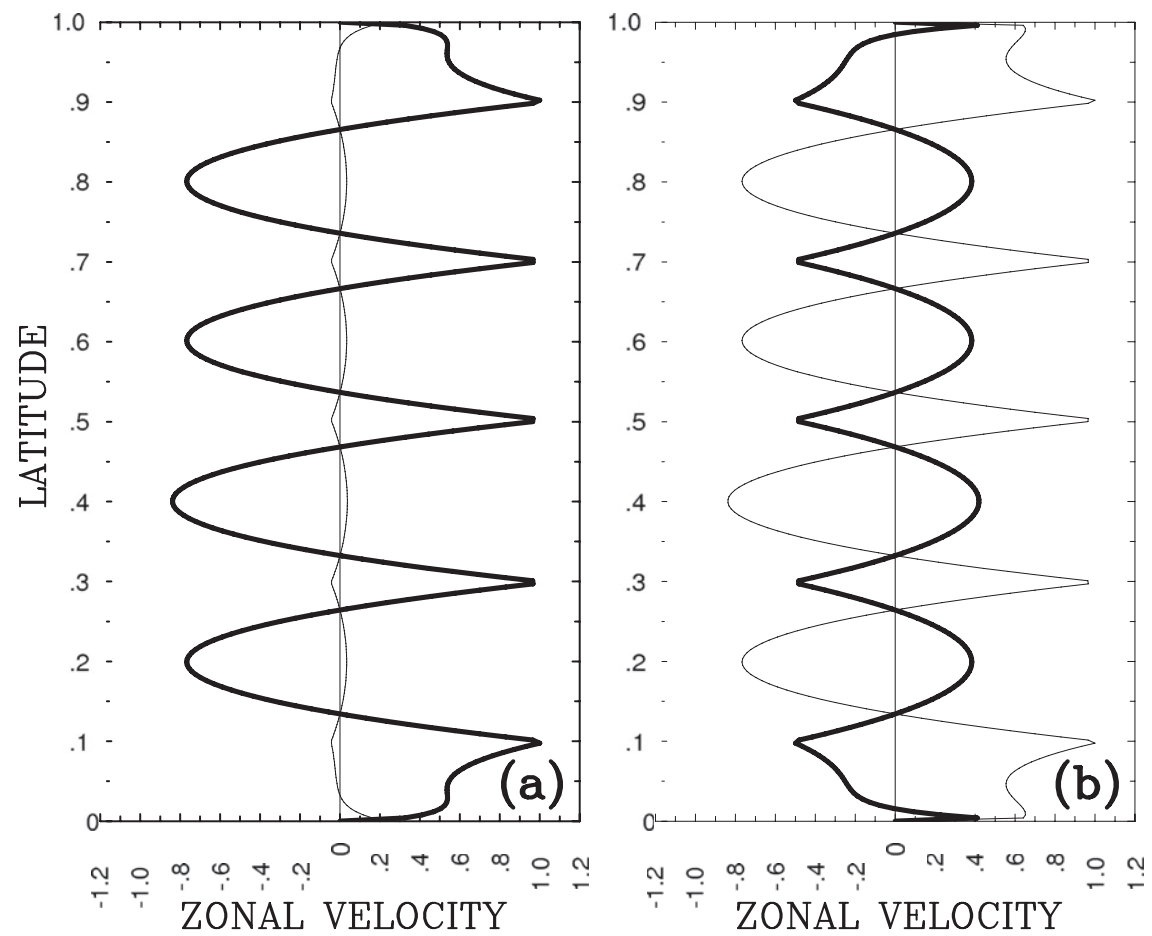

FIG. 7. Zonal velocity profiles corresponding to the ideal PV staircases. These velocities are in sharp contrast to velocities of the actual solutions of the flow dynamics (Figs. 2 and 3). Isopycnal-layer velocities of the (a) EB and (b) WB flow reference solutions. Upper- and deepocean velocity profiles are normalized by their peak values and shown with thick and thin curves, respectively.

for compatibility and comparison with numerous previous studies of the multiple jets in barotropic models (section 1a). Thus, $L_{j}$ is diagnosed from the following relationships:

$$
L_{j}=\frac{2 \pi}{k_{j}}, \quad 2 \int_{0}^{k_{j}} E(k) d k=\int_{0}^{\infty} E(k) d k .
$$

The above definition ${ }^{5}$ is different from the one used in P93 and Berloff (2005), where $k_{j}$ corresponds to the maximum of $E(k)$. This is motivated by the fact that the spectrum typically has two extrema: the new definition removes potential discontinuities from the functional dependency of $k_{j}$ on parameters of the problem.

An important length scale of the problem is the Rhines scale

$$
L_{r}=\left(\frac{U}{\beta}\right)^{1 / 2}
$$

\footnotetext{
${ }^{5}$ When all jets are clearly visible and therefore can be easily counted, $L_{j}$ is roughly equivalent to the channel width divided by the number of the jets.
}

where $U$ is some velocity scale that characterizes transient fluctuations of the flow. There is ambiguity in choosing $U$; nevertheless, in $\mathrm{P} 93$ it is argued that with $U$ chosen as the square root of the eddy kinetic energy, the meridional width of the multiple zonal jets scales with (20) - that is, $L_{j} \sim L_{r}$. Here, we make the same choice of $U$. (An alternative choice of $U$ might be based on the barotropic-mode or upper-ocean velocity variances.)

The dependence of $L_{j}$ on the background shear is obtained by calculating two sets of solutions that have either eastward or westward $U_{1}$ (and $U_{2}=0$ ). All other parameters are as in the reference solutions. Overall, $L_{j}$ increases with the shear, but the underlying relationship is not simple. At least three different ${ }^{6}$ solution branches, obtained by starting the model from different initial conditions, are found in each set of the solutions (Fig. 8a). These branches represent multiple equilibria of the flow characterized by different numbers of the jets. By plotting the ratio $r=L_{j} L_{r}^{-1}$ against $U_{1}$, we find

\footnotetext{
${ }^{6}$ To our knowledge, multiple solutions have been reported only once, with a barotropic multiple-jet model (Danilov and Gurarie 2004).
} 

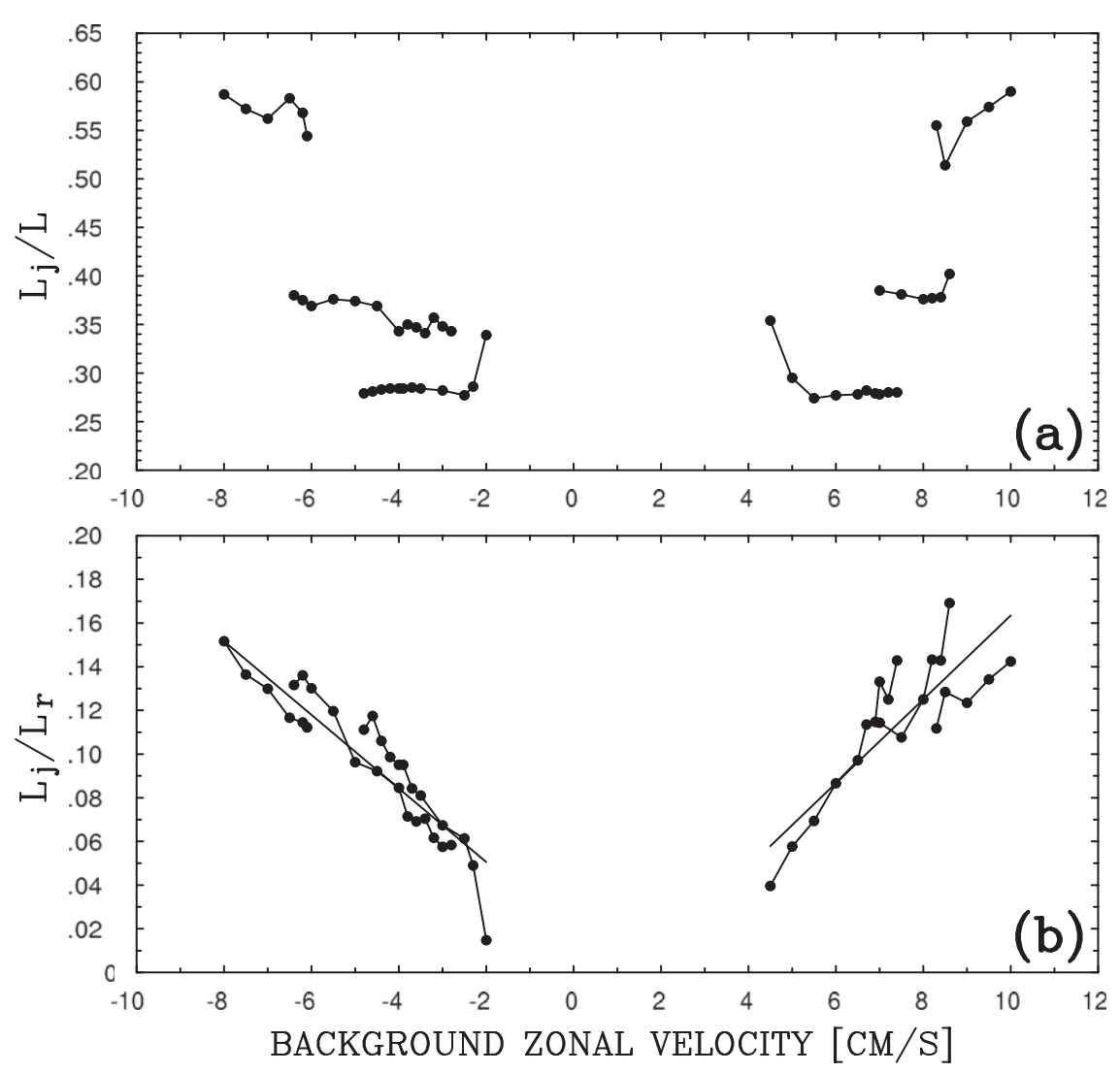

FIG. 8. Dependence of $L_{j}$ on the background flow velocity. Ratio of (a) $L_{j}$ to the channel width and (b) $L_{j}$ to the Rhines scale $L_{r}$. Multiple solution branches are connected with continuous curves; straight lines in (b) indicate linear best square fit.

that $L_{j}$ does not scale with $L_{r}$, even on the individual branches (Fig. 8b). The Rhines scaling (20) would imply that $r$ is constant but this is, apparently, not the case, because $r$ changes by a factor more than 5 over the explored range of $U_{1}$. A better scaling estimate would be $r=c_{1}+c_{2} U_{1}$, where the constant coefficients can be estimated from the EB- and WB-flow linear fits (Fig. 8b).

The dependence of $L_{j}$ on $\beta$ is studied by varying $\beta$ around its reference value of $2 \times 10^{-11} \mathrm{~m}^{-1} \mathrm{~s}^{-1}$ (Fig. 9). We find that $L_{j}$ is minimal on the plateau that includes the reference value of $\beta$, and it gradually increases away from it. The Rhines scaling (20) is generally not confirmed because $r$ is not nearly constant. However, in the EB flow, for $\beta$ in the range between 1 and $2 \times$ $10^{-11} \mathrm{~m}^{-1} \mathrm{~s}^{-1}$, the Rhines scaling is approximately confirmed, which is consistent with P93, where the same range of $\beta$ is explored. For smaller values of $\beta$, the flow solutions are dominated by very energetic eddies by a few weak and broad jets in the interior of the channel and by intense and narrow baroclinic eastward jets near the zonal boundaries. This flow regime is driven by the eddies that efficiently mix buoyancy in the meridional direction and thus significantly flatten the background isopycnals, so that the time-mean flow becomes less supercritical.

The variations of the bottom friction parameter $\gamma$ reveal that, for both background flow directions, $L_{j}$ moderately decreases when $\gamma$ increases from zero to more realistic values (Fig. 10a). In the WB flow, this tendency reverses at $\gamma \approx 10^{-7} \mathrm{~s}^{-1}$ (i.e., spin-down time of about four months). The increase of $L_{j}$ at larger values of $\gamma$ is associated with the fading of the barotropic component of these jets as a result of the intense bottom friction and is in disagreement with the Rhines scaling. This large- $\gamma$ regime is dominated by the near-boundary jets. In the EB flow, $L_{j}$ drops by $30 \%$ as $\gamma$ increases from zero to about $10^{-8} \mathrm{~s}^{-1}$ (i.e., spin-down time of about 1-2 weeks); after that, it remains largely insensitive to further increase of 7 . In the range $10^{-7} \mathrm{~s}^{-1}<\gamma<4 \times$ $10^{-7} \mathrm{~s}^{-1}$, as has been explored in $\mathrm{P} 93, L_{j}$ is approximately proportional to $L_{r}$. This implies that our results are consistent with P93, who reports Rhines scaling in the narrower range of parameters. However, we do not confirm Rhines scaling for smaller values of $\gamma$ and in the WB flow, overall. 

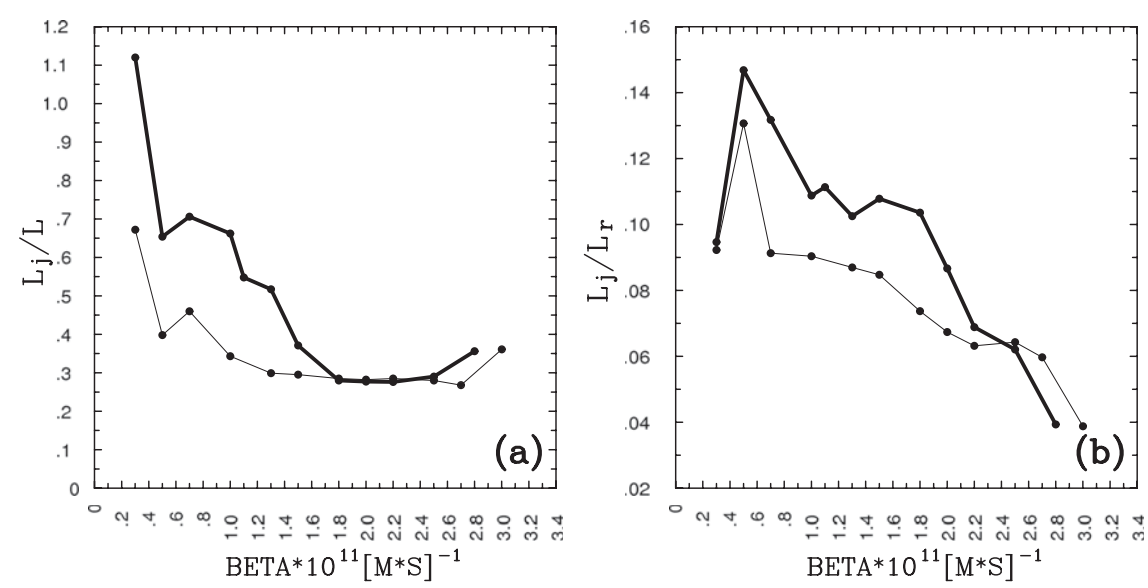

FIG. 9. Dependence of $L_{j}$ on $\beta$. (a) Ratio of $L_{j}$ to the channel width is shown for EB (thick curve) and WB (thin curve) flow solutions. (b) Ratio of $L_{j}$ to $L$, for the same set of solutions.

We also varied eddy viscosity $\nu$ from the half of the reference value of $100 \mathrm{~m}^{2} \mathrm{~s}^{-1}$ to the value at which the flow is linearly stable (Fig. 10b). The exploration of very large Reynolds numbers corresponding to even lower values of $\nu$ is left for the future because it requires massive computations on a finer horizontal grid needed for resolving finer length scales of the eddies. Overall, $L_{j}$ increases with $\nu$ more steeply in the WB flow. There is a sign of convergence of $L_{j}$ at the values of $\nu$ smaller and about the reference value of $100 \mathrm{~m}^{2} \mathrm{~s}^{-1}$, suggesting the robustness of the multiple-jet configuration. Rhines scaling for the sets of solutions with variable $\nu$ is not confirmed as, over the explored range of $\nu, r$ decreases by a factor of 4 in the WB flow and by about $40 \%$ in the EB flow (Figs. 11 and 12).

Finally, we explored the sensitivity of the jets to variations of the stratification in the two-layer model by varying Rossby deformation radius $\mathrm{Rd}_{1}$ from 10 to
$35 \mathrm{~km}$ and the ratio of the layer depth $H_{1} / H_{2}$ from 0.15 to 1.0. We found that the WB flow jets are nearly insensitive to these variations, whereas in the EB flow the jet width moderately increases for larger ratio and for smaller $\mathrm{Rd}_{1}$, in accordance with the flow becoming more supercritical.

\section{Nonlinear dynamics}

In this section, the dynamical effects of the eddies on the multiple jets are analyzed and quantified in terms of the eddy forcing and its components. A theoretical explanation of these findings is presented in Berloff et al. (2009).

\section{a. Formulation of the eddy forcing and its components}

In the channel, the time-mean PV balance is between eddy forcing and dissipation. The key dynamical question

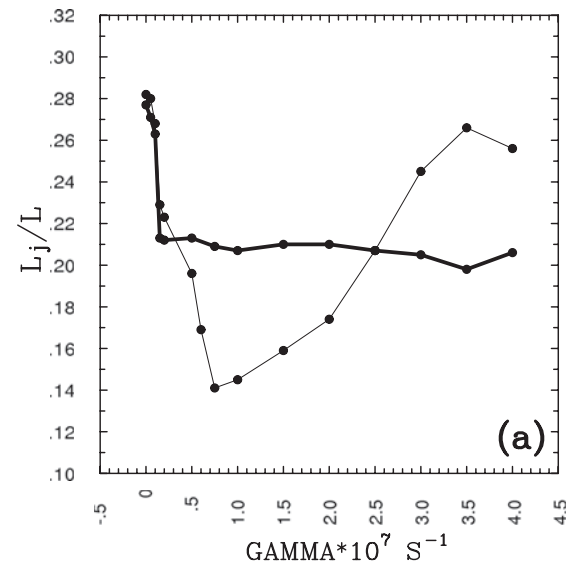

FIG. 10. Dependence of $L_{j}$, on (a) $\gamma$ and (b) $\nu$. Individual dots are connected with curves, except when straight line is least square fitted in (b), where thick and thin curves correspond to the EB and WB flow solutions, respectively. 

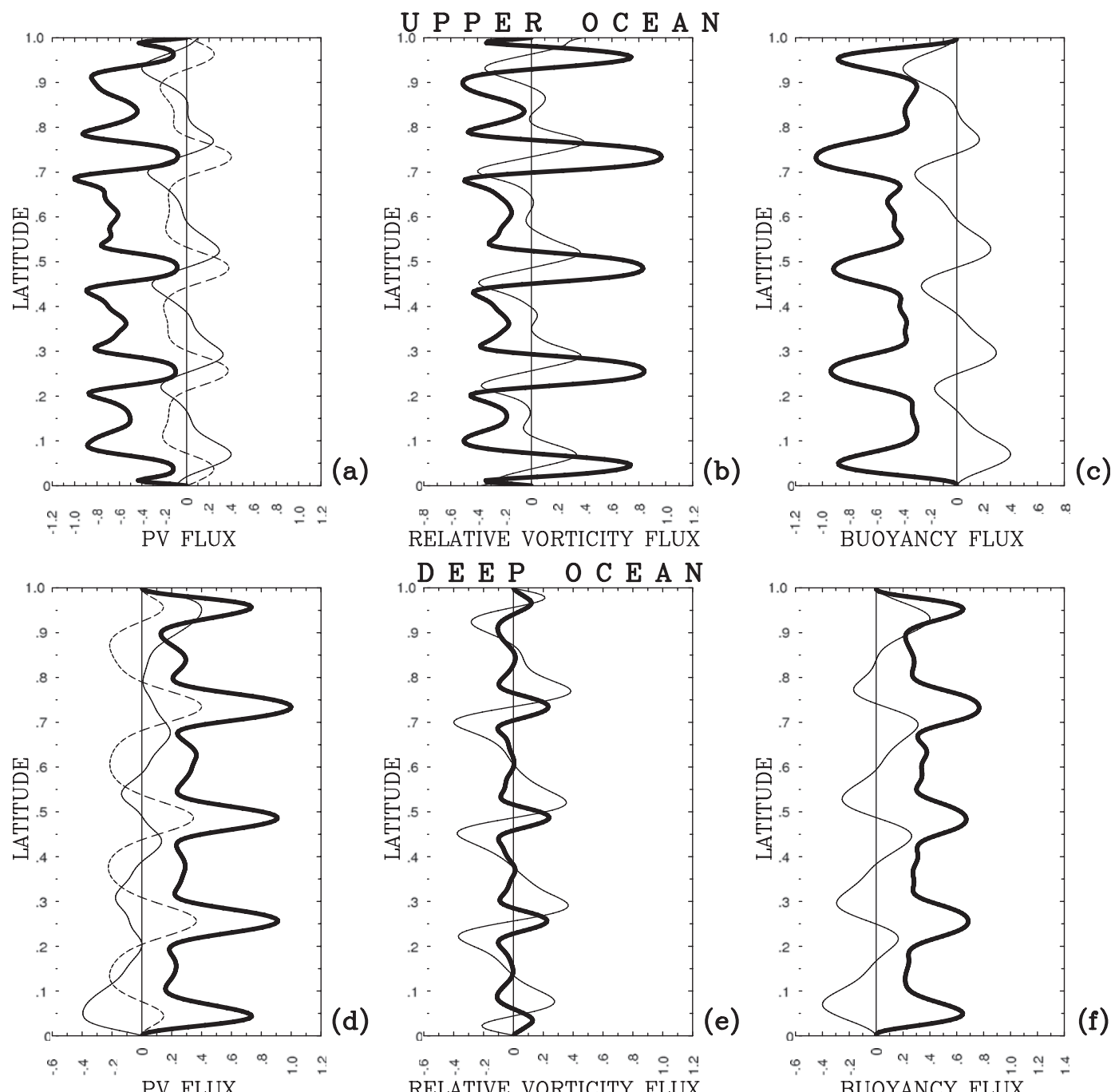

(f)

FIG. 11. Time-mean meridional eddy fluxes of PV and its components in the reference EB solution. (top) Fluxes of (a) PV, (b) relative vorticity, and (c) buoyancy in the upper ocean. (bottom) The same quantities as (a) but for the deep ocean. In each panel, the flux (thick curve) is normalized by the maximum value of the corresponding full PV flux. The corresponding profiles of the time-mean PV and its components are shown with thin curves, and the profiles of the time-mean zonal velocity are shown with dashed curves but only in (a),(d) (both of these quantities have arbitrary units).

addresses the composition of the eddy forcing in terms of the relative vorticity and buoyancy components. The analysis of the eddy forcing in terms of the vertical-mode interactions illuminates the role of baroclinic dynamics and permits the comparison with the barotropic and equivalent-barotropic models (section 1a).

The vertical-mode velocity streamfunctions and potential vorticities are related to the corresponding isopycnallayer quantities as

$$
\begin{aligned}
& \phi^{(i)}(t, x, y)=\theta_{i j} \psi_{j}(t, x, y) \quad \text { and } \\
& p^{(i)}(t, x, y)=\theta_{i j} q_{j}(t, x, y),
\end{aligned}
$$

where the superscript $i$ is the mode index, summation over $j=\overline{1, N}$ ( $N$ is the number of the active isopycnal layers/vertical modes) is assumed, and $\theta_{i j}$ is the transformation matrix calculated from the stratification parameters (e.g., McWilliams 2006). The corresponding inverse transformation matrix is

$$
\omega_{i j}=\theta_{i j}^{-1}
$$

In terms of the vertical modes, the relationship (5)-(7) that connects the PV and velocity streamfunction fields is diagonalized as follows: 

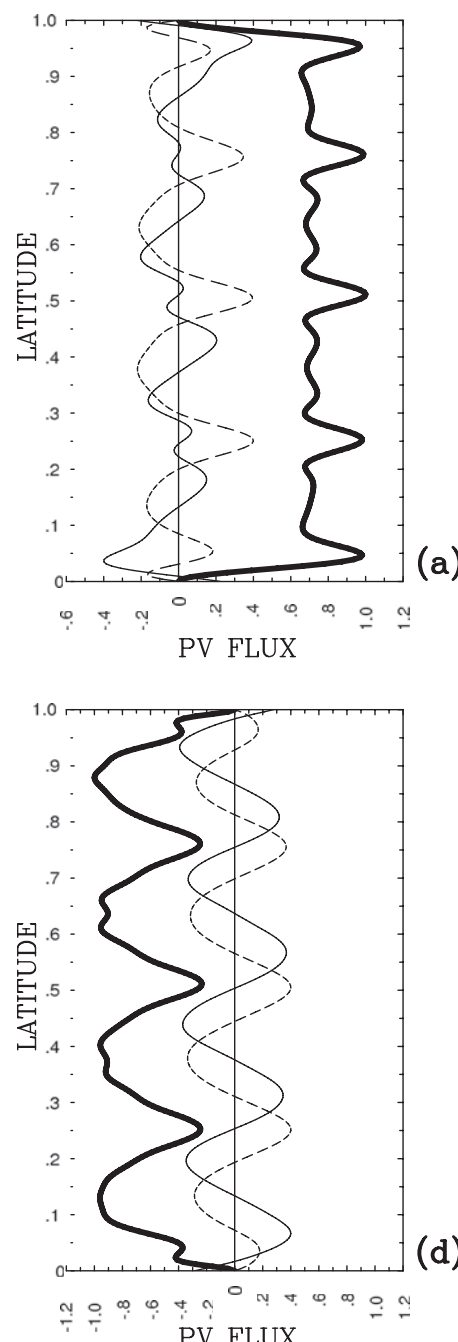

(d)

FIG. 12. The same as in Fig. 11, but for the reference WB solution.

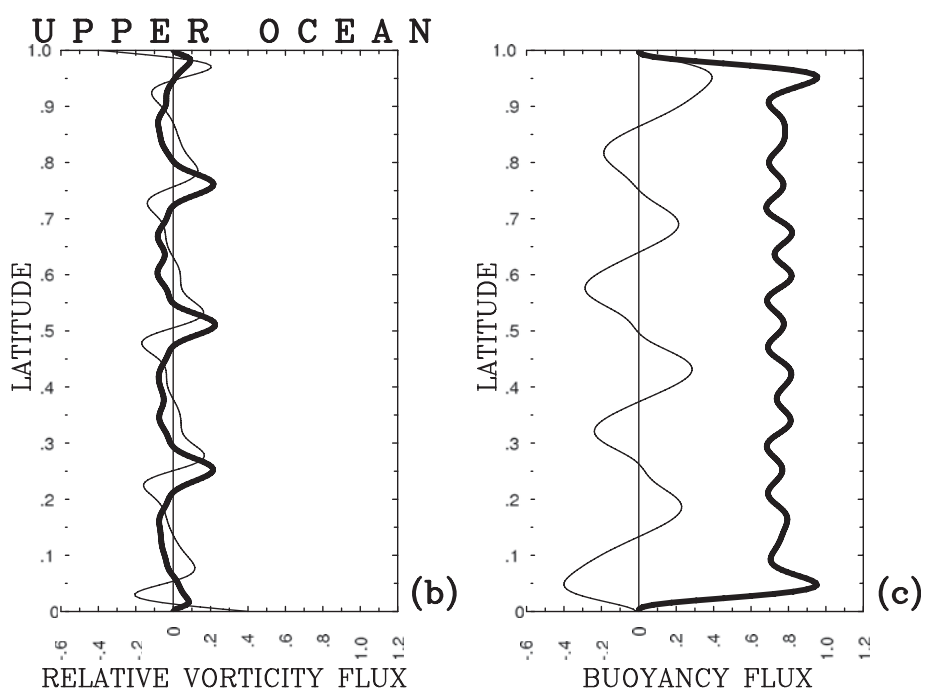

D E E P O C E A N
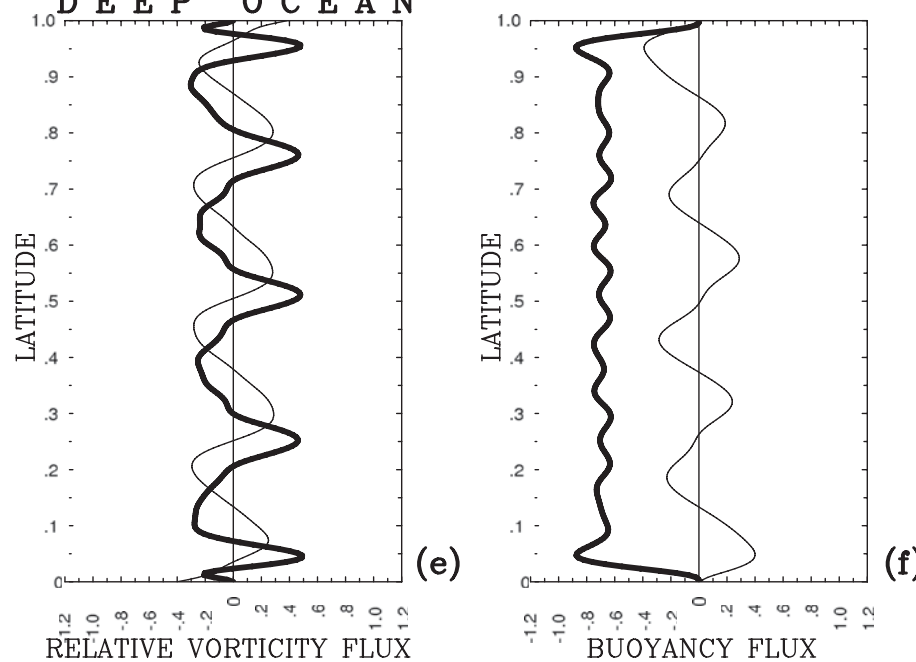

$\Theta_{i j}^{(2)}=0 \quad$ if $\quad i=j=1, \quad$ or $\quad i=1$,

$j=3, \quad$ or $i=3, \quad j=1, \quad$ and

$\Theta_{i j}^{(3)}=0 \quad$ if $\quad i=j=1, \quad$ or $\quad i=1$,

$j=2, \quad$ or $\quad i=2, \quad j=1$.

Eddy forcing is also projected on the vertical modes

$$
\begin{aligned}
F^{(i)}(x, y) & \equiv-\Theta_{k l}^{(i)} \overline{J\left[\phi^{\prime(k)}, p^{\prime(l)}\right]}, \\
& \equiv \sum_{k, l}^{N} F_{k l}^{(i)}=\sum_{k, l}^{N}\left[F_{R_{k l}^{(i)}}^{(i)}+F_{B_{k l}}^{(i)}\right] .
\end{aligned}
$$

We focus on the relative contributions of individual verticalmode interactions to eddy forcing; the corresponding matrix $\rho_{k l}^{(i)}$ has elements that sum up to the unity:

$$
\Theta_{i j}^{(1)}=0 \quad \text { if } \quad i \neq j
$$



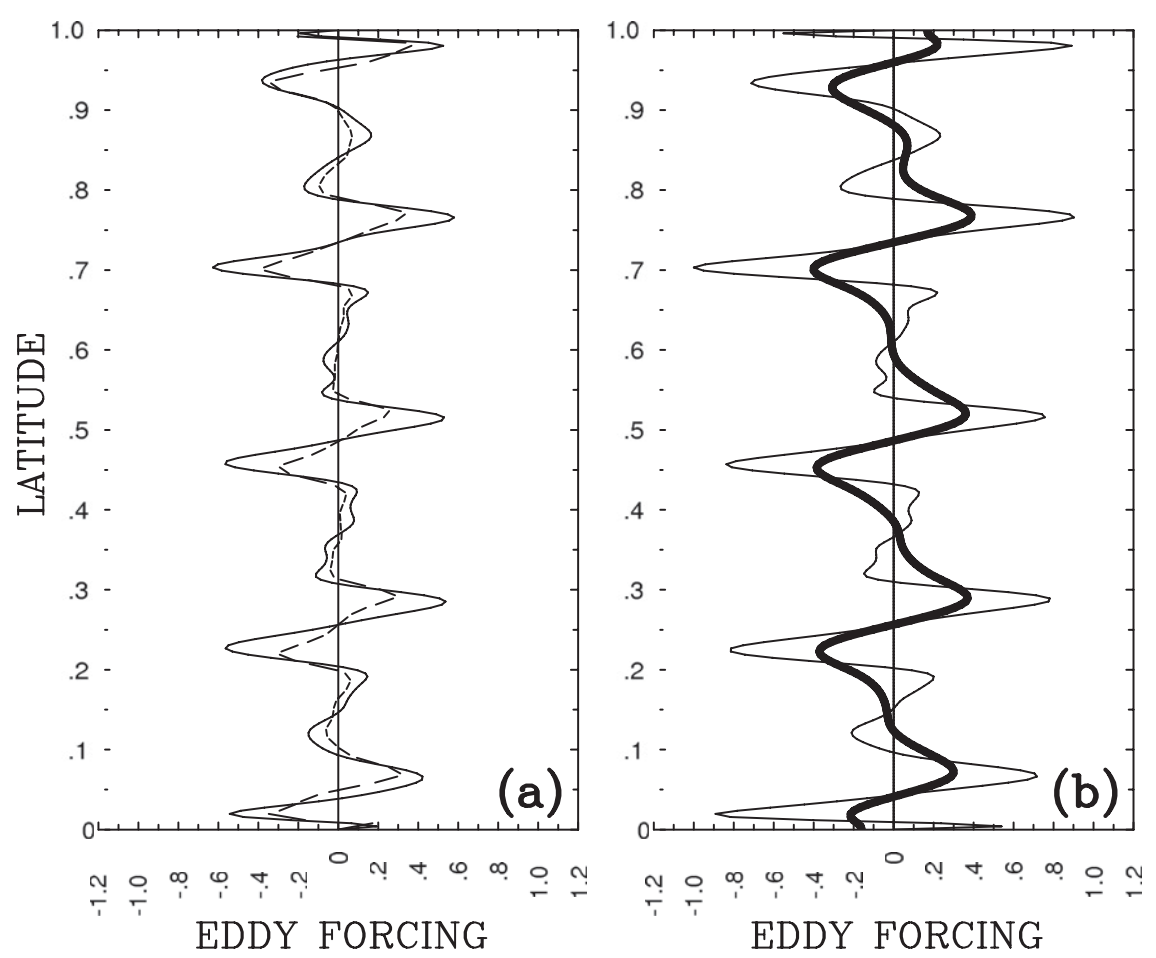

FIG. 13. Barotropic eddy forcing and its components. (a) Barotropic-barotropic (continuous) and baroclinic-baroclinic (dashed curve) eddy forcing components of the reference, two-layer EB flow solution. (b) Full eddy forcing (thin) is shown along with the time-mean barotropic PV component (thick curve). The eddy forcing itself and its components are normalized by the maximum value of the eddy forcing; the barotropic PV is shown with arbitrary units.

$$
\rho_{k l}^{(i)}=\frac{\iint F_{k l}^{(i)} F^{(i)} d x d y}{\iint F^{(i)^{2}} d x d y}
$$

The elements of the matrix are also decomposed into the Reynolds stress $\rho_{R_{k l}}^{(i)}$ and form stress $\rho_{B_{k l}}^{(i)}$ forcing components. Positive/negative values of $\rho^{(i)}$ indicate that the corresponding component of the eddy forcing maintains/resists the $i$ th vertical component of the jets. The magnitude of $\rho^{(i)}$ quantifies the contribution of the corresponding eddy forcing component to the total eddy forcing.

\section{b. Analysis of the eddy forcing}

With the eddy forcing analysis presented in this section, we capture the essentials of the eddy/large-scale interactions driving the jets. In particular, we analyze vertical-mode interactions and find Reynolds stress and form stress contributions to the eddy forcing.

Both in the EB and WB flows, the barotropic eddy forcing is concentrated around the cores of the eastward jets (Fig. 13). The corresponding barotropic-barotropic $F_{11}^{(1)}$ and baroclinic-baroclinic $F_{j j}^{(1)}(j>1)$ eddy forcing components are equally important and have similar patterns (Fig. 13). The values of $\rho^{(1)}$ (Table 2) suggest the following conclusions. First, the barotropic component of the multiple jets cannot be accurately modeled with purely barotropic dynamics driven by small-scale random fluctuations, which are often thought of as the approximation for the baroclinic eddy forcing (section 1a). If the first baroclinic mode is not explicitly taken into account, then the external forcing has to include some meridionally varying structure, which accounts for the baroclinic eddy effects. Second, the barotropic eddy forcing components corresponding to the barotropic-barotropic and baroclinic-baroclinic interactions are positively correlated; that is, they work together rather than compete with each other. Third, the comparison of the two- and three-layer barotropic dynamics

TABLE 2. Vertical-mode representation of the eddy forcing in the barotropic zonal-channel dynamics. The nonzero $\rho$ terms are shown for the three- and two-layer (in brackets) multiple-jet reference solutions.

\begin{tabular}{lccc}
\hline & $\rho_{11}^{(1)}$ & $\rho_{22}^{(1)}$ & $\rho_{33}^{(1)}$ \\
\hline EB & $+0.47(+0.63)$ & $+0.43(+0.37)$ & +0.10 \\
WB & $+0.63(+0.72)$ & $+0.34(+0.28)$ & +0.03 \\
\hline
\end{tabular}


TABLE 3. Same as in Table 2, but for the first baroclinic dynamics. The columns and rows correspond to the first and second indices of $\rho_{k l}^{(2)}$, respectively. The $\rho$ terms with nonzero eddy buoyancy forcing are split into the Reynolds stress and form stress forcing components indicated by $[\mathrm{R}]$ and $[\mathrm{B}]$, respectively. The two-layer $\rho$ terms are shown in brackets. Empty sections of the table correspond to $\rho_{k l}^{(2)}=0$.

1

1

3

\author{
$\mathrm{WB}:+1.43=+0.61[\mathrm{R}]+0.82[\mathrm{~B}]$ \\ $(\mathrm{WB}:+1.81=+0.70[\mathrm{R}]+1.11[\mathrm{~B}])$ \\ $\mathrm{EB}:+0.27=+0.32[\mathrm{R}]-0.06[\mathrm{~B}]$ \\ $(\mathrm{EB}:-0.28=+0.19[\mathrm{R}]-0.47[\mathrm{~B}])$ \\ WB: +0.02 \\ (WB: +0.09$)$ \\ EB: +0.07 \\ (EB: +0.50$)$ \\ WB: $-0.18=-0.07[\mathrm{R}]-0.11[\mathrm{~B}]$ \\ $\mathrm{EB}:+0.01=-0.02[\mathrm{R}]+0.03[\mathrm{~B}]$
}

$\mathrm{WB}:+0.58=+0.10[\mathrm{R}]+0.48[\mathrm{~B}]$

EB: $+0.14=+0.05[\mathrm{R}]+0.09[\mathrm{~B}]$

WB: +0.03

$\mathrm{EB}:+0.02$ suggests that the contribution of the second baroclinic mode is relatively unimportant in the WB flow, but it is moderately important in the EB flow. In the EB flow, the barotropic-barotropic interaction accounts for nearly three-quarters of the total two-layer eddy forcing; however, in the three-layer model it accounts for less than one-half of the eddy forcing. This suggests that the relative contribution of the barotropic-barotropic interaction decreases with better vertical resolution.

The baroclinic dynamics is more complex than the barotropic dynamics because of the many eddy forcing terms involved. The first and second baroclinic-mode $\rho$ matrices are given in Tables 3 and 4 , respectively, and the first baroclinic $\rho$ matrix is calculated for both the two- and three-layer models.

The meridional profiles of the baroclinic eddy forcing and some of its components are shown in Fig. 14. The analysis of the $\rho$ matrices and the corresponding meridional profiles of the eddy forcing components suggests the following: In the EB and WB flows, barotropicbaroclinic and baroclinic-baroclinic interactions are equally important, and they maintain the jets. The second baroclinic mode is important for both background flow directions. Apart from these aspects, the EB and WB flow dynamics are rather different, and their differences are summarized next.

In the EB flow, the baroclinic component of the jets is maintained/resisted by the Reynolds/form stress eddy forcing component (e.g., as in P93). In the three-layer model, the resisting role of the form stress forcing shifts to the second-mode baroclinic dynamics, and this role is due to interactions with the second baroclinic mode. The resisting role of the form stress forcing indicates the baroclinic instability of the jets. On the other hand, advections of the barotropic and first-baroclinic relative vorticities by the first baroclinic mode (i.e., the corresponding Reynolds stress forcings) maintain the jets, which can be viewed as a "negative" eddy viscosity effect (e.g., Starr 1968).
In the WB flow, both in the two- and three-layer models, the Reynolds stress and form stress eddy forcings act opposite to what they do in the EB flow. The baroclinic components of the jets are maintained mostly by the corresponding form stress forcings. The Reynolds stress forcing associated with advection of the barotropic relative vorticity by the baroclinic modes acts against the form stress forcing. ${ }^{7}$ Thus, the eddies act to destroy the jets through the "positive" eddy viscosity effect, but they maintain the jets through the locally converging eddy buoyancy flux that properly tilts isopycnals. Although, the ultimate source of the energy is the baroclinic tilt of the interface, the eddies maintain the jets by locally releasing their available potential energy back into the time-mean flow; in this sense, this mechanism is opposite to the baroclinic instability. Next, we find that advection of the baroclinic relative vorticity by the baroclinic modes is relatively unimportant and that cross-interactions of the baroclinic modes are more important than their self-interactions. Finally, we notice that the WB eddy forcing is characterized by the meridional high-frequency harmonic, which is a natural outcome of the quadratic nonlinearity.

We also analyzed the dynamics for the cases of moderately supercritical flows with equal layer depths and with $U_{1}$ equal to either 6.0 or $-2.7 \mathrm{~cm} \mathrm{~s}^{-1}$. We do not find strong barotropization of the flow, as predicted by Smith and Vallis (2002); instead, we find noticeable qualitative changes only in the EB flow and only in the baroclinic-mode dynamics. There, we find that the barotropic mode is twice as strong as the baroclinic mode and that there is the corresponding weakening of the baroclinic-baroclinic interactions at the expense of the barotropic-baroclinic interactions.

\footnotetext{
${ }^{7}$ The relatively weak, though positive, role of the Reynolds stress forcing in maintaining a single jet on the WB flow is reported in Ivchenko et al. (1997).
} 
TABLE 4. Same as in Table 3, but for the second baroclinic dynamics.

\begin{tabular}{llll}
\hline \hline \multicolumn{1}{c}{1} & \multicolumn{1}{c}{2} & \multicolumn{1}{c}{3} \\
\hline 1 & & WB: $+1.03=+0.24[\mathrm{R}]+0.80[\mathrm{~B}]$ \\
& & EB: $-0.84=+0.22[\mathrm{R}]-1.06[\mathrm{~B}]$ \\
2 & & WB: $+0.36=+0.06[\mathrm{R}]+0.30[\mathrm{~B}]$ \\
& & WB: -0.01 & EB: $+1.11=+0.29[\mathrm{R}]+0.82[\mathrm{~B}]$ \\
& EB: +0.38 & WB: +0.01 \\
& WB: -0.24 & WB: $-0.16=-0.09[\mathrm{R}]-0.07[\mathrm{~B}]$ & EB: -0.07 \\
\hline
\end{tabular}

\section{Role of meridional boundaries}

In this section, we study the importance of the meridional boundaries by adding them to the model and by analyzing the corresponding solutions. To keep the analysis as simple as possible, we consider a basin (equivalent to half of the channel) in which the background flow is the same as in the previous sections; that is, we allow the background flow to go unaltered by the meridional boundaries. Those boundaries, however, are used to constrain the perturbations to that flow to satisfy zero normal velocity conditions. We, therefore, consider the jets in the middle of oceanic gyres, away from the meridional boundaries. This intermediate way station between a complete basin model and the zonally periodic channel isolates the effects of the meridional boundaries and keeps the basic problem simple.

For the closed-basin reference solutions, we use the values of $U_{1}=6.5$ and $-2.5 \mathrm{~cm} \mathrm{~s}^{-1}$ that yield the clearest multiple-jet patterns (Fig. 15). The jets are substantially weaker than those in the channel despite the equally strong eddies. Also, the jets do not have the noticeable east-west asymmetry of the zonal velocity that we found in the channel, and we could not find multiple flow equilibria. Finally, the tendency toward the PV staircasealready weak in the zonal channel-is even weaker in the closed basin. All these properties are also characteristic of the multiple jets studied in the North Atlantic GCM (KBP09).

Another important aspect of the closed-basin dynamics is the excitation of the weakly damped, large-scale, second baroclinic basin mode in the WB flow (e.g., Berloff 2005). The excited basin mode propagates to the west on the interannual time scale (i.e., with basin-crossing time of the second baroclinic mode) and explains several properties of the solution. First, in the middle of the basin, the instantaneous jets are efficiently averaged out, in the timemean sense, by the meridional fluctuations associated with the propagating basin mode. Second, in the time-mean baroclinic component of the flow, there are well-defined, large-scale cyclonic and anticyclonic recirculations (associated with the several jets) near the northern and southern basin boundaries, respectively. These features are driven by the nonlinear self-interactions of the mode
(Berloff 2005). In the EB flow, the situation is different because the least damped basin mode has to propagate against the background flow, which shortens its zonal length scale and thus makes it more damped.

The strong zonal inhomogeneity of the jets is a robust feature of the WB flow: the jets are attached to the western boundary and extend over three-quarters and one-half of the basin in the two- and three-layer models, respectively. In the WB flow, the zonal inhomogeneity of the jets is stronger and the jets are also more latent. These are qualitatively consistent with the North Atlantic simulation (KBP09).

We explored how the width of the jets $L_{j}$ depends on the parameters of the problem by finding several sets of flow solutions. Overall, we found general similarities with the channel (section 4), except that in the closed basin the width of the jets is typically $20 \%-30 \%$ shorter. We also found that the dynamical balances in the interior of the closed basin and in the zonal channel are qualitatively similar (section 5), but in the basin the eddy forcing is noticeably weaker. The closed-basin barrier/mixer contrasts from the kinematical analysis (as in section 3) are found to be much weaker than in the channel.

\section{Conclusions and discussion}

Multiple alternating zonal jets observed in the ocean are studied with an idealized quasigeostrophic zonalchannel model, with the supercritical, zonal background flow imposed. In the model, the jets are maintained by the mesoscale eddies generated by the flow instabilities. On the one hand, we continue research line carried out by the atmospheric community interested in the multiple zonal jets on Jupiter. On the other hand, we are guided by the recent discovery of multiple zonal jets in the oceans and by the jets reported in the eddy-resolving simulations.

Observations and comprehensive GCMs (section 1a) suggest that the multiple zonal jets have equally important barotropic and baroclinic components. A similar conclusion has been drawn by Thompson and Young (2007). What is the minimal number of the vertical modes that have to be taken into account? To answer this question, we systematically studied the interactions 

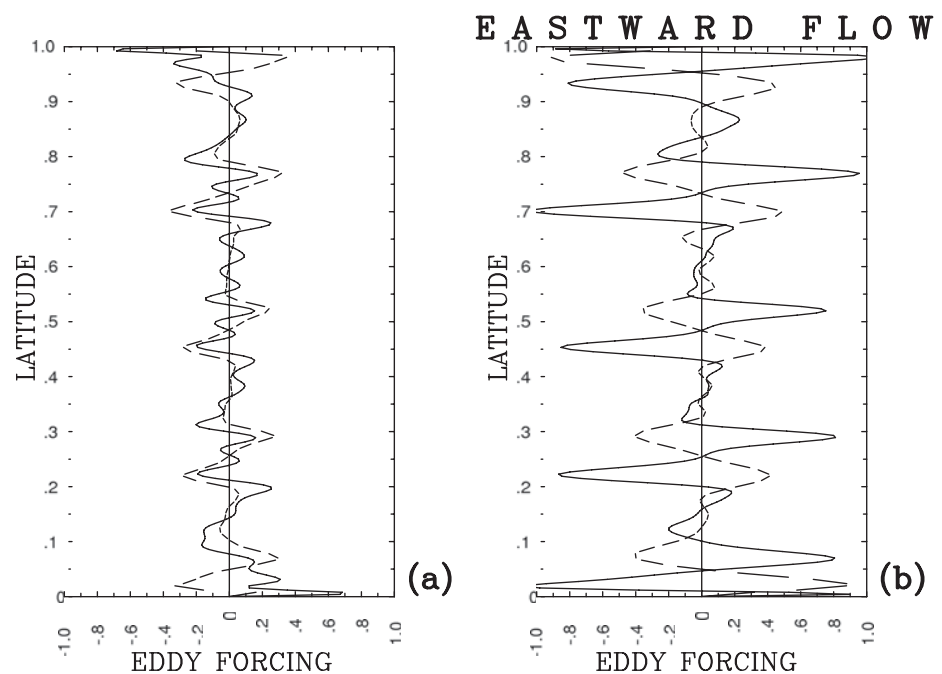

(a)

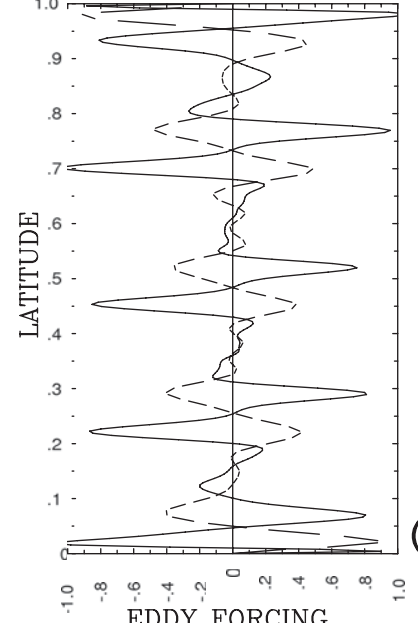

EDDY FORCING
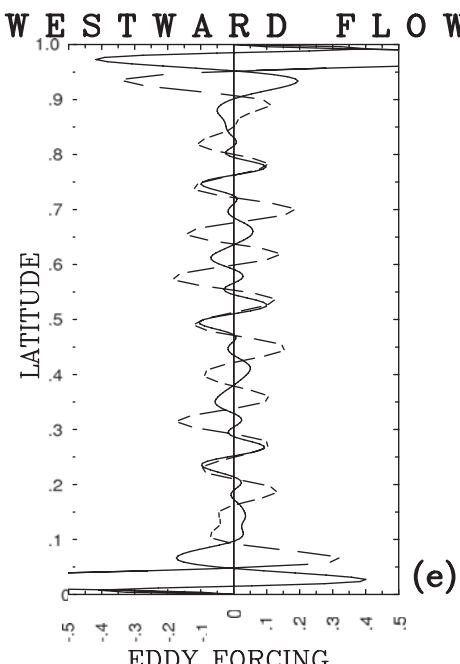
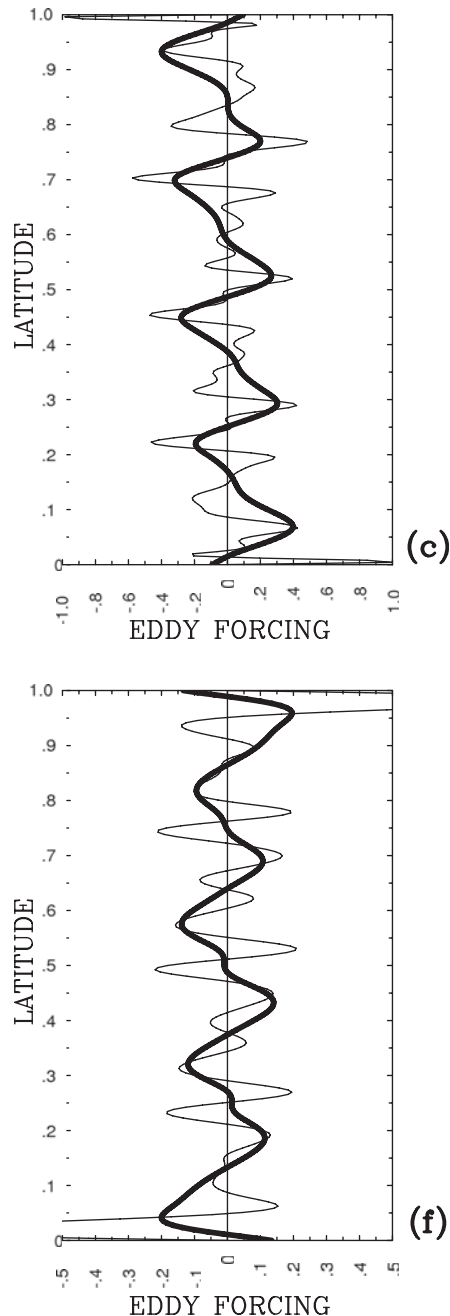

(f)

FIG. 14. Baroclinic eddy forcing and its components. (a) Barotropic-baroclinic (thin) and baroclinic-baroclinic (dashed curve); (b) relative-vorticity (thin) and buoyancy (dashed curve) eddy forcing components of the reference, two-layer EB flow solution; (c) full eddy forcing (thin) is shown along with the time-mean baroclinic PV component (thick curve). The eddy forcing itself and its components are normalized by the maximum value of the eddy forcing; the baroclinic PV is shown with arbitrary units. (bottom) The same quantities as in (a)-(c), but for the WB flow solution.

between the vertical modes. Our results suggest that for capturing the qualitative dynamics, the two-layer model is the minimal one and for more accurate quantitative predictions, the second baroclinic mode and, perhaps, even higher modes have to be taken into account.

We find that the barotropic component of the jets is maintained by both barotropic-barotropic and baroclinicbaroclinic time-mean interactions. Thus, the prediction of the barotropic component of the jets from a purely barotropic model in which effects of the higher modes are approximated by the small-scale random forcing (e.g., as in a number of the studies discussed in section 1a) can be problematic. This is because the barotropic jets are largely driven by the baroclinic-mode Reynolds stress forcing.
Similarly, the prediction of the baroclinic component of the jets from the randomly forced equivalentbarotropic model is likely to be inaccurate because of the missing contribution from the barotropic eddies. In particular, in the eastward background flow the baroclinic component of the jets is maintained by the barotropic-baroclinic interactions, whereas in the westward background flow both the barotropic-baroclinic and baroclinic-baroclinic interactions are found to be important. Also, not only the first but also the second baroclinic mode is found to be important for maintaining the baroclinic component of the jets; the barotropic component of the jets is maintained mostly by the barotropic and first baroclinic modes. 


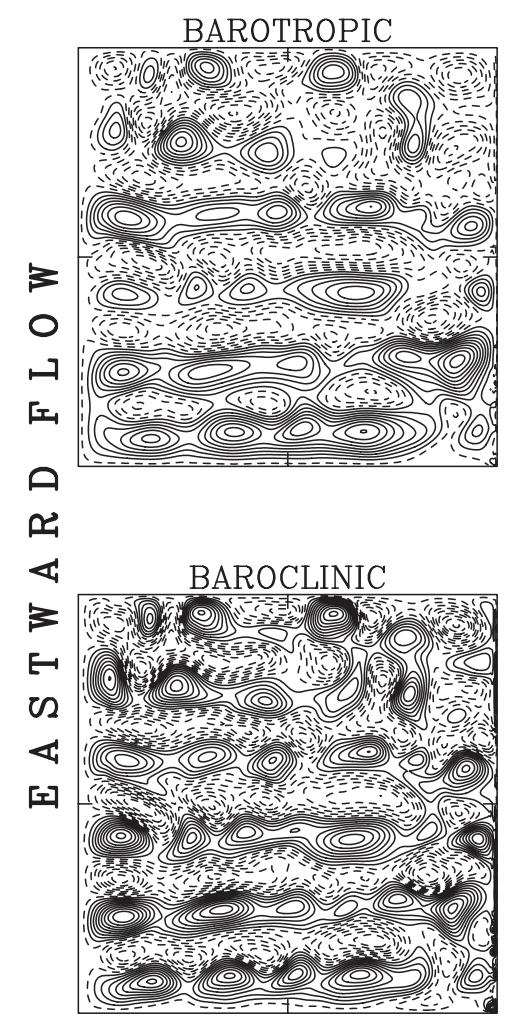

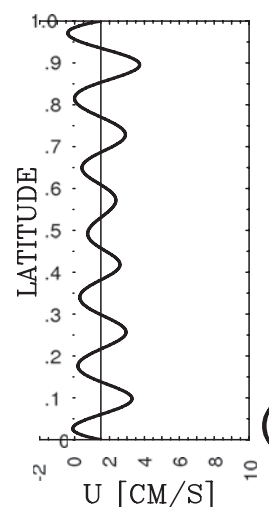

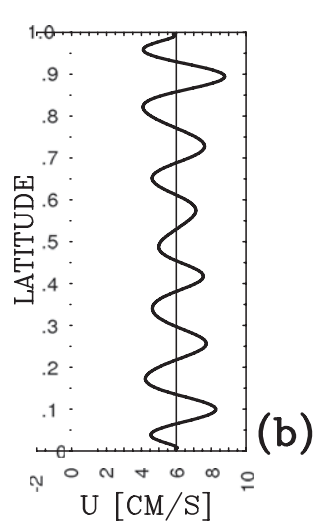

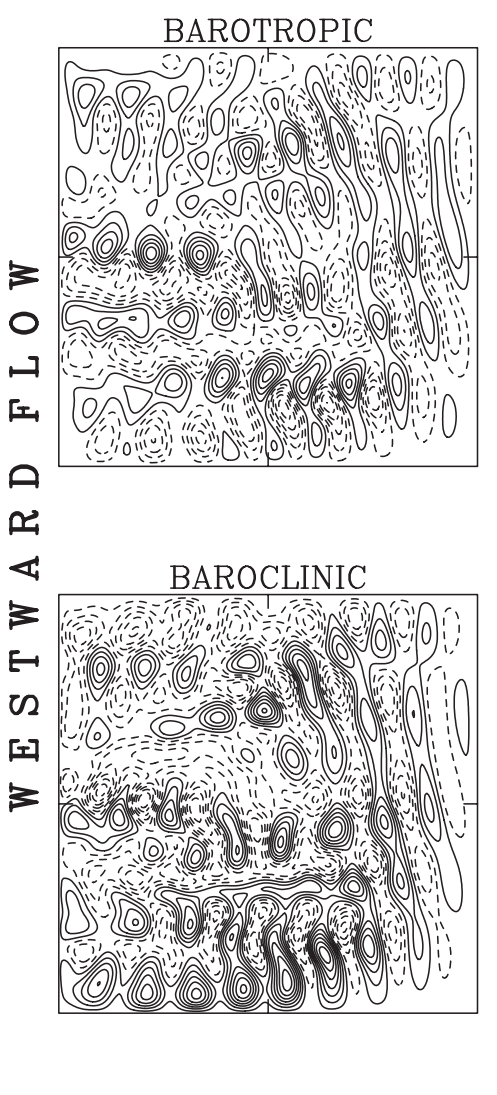
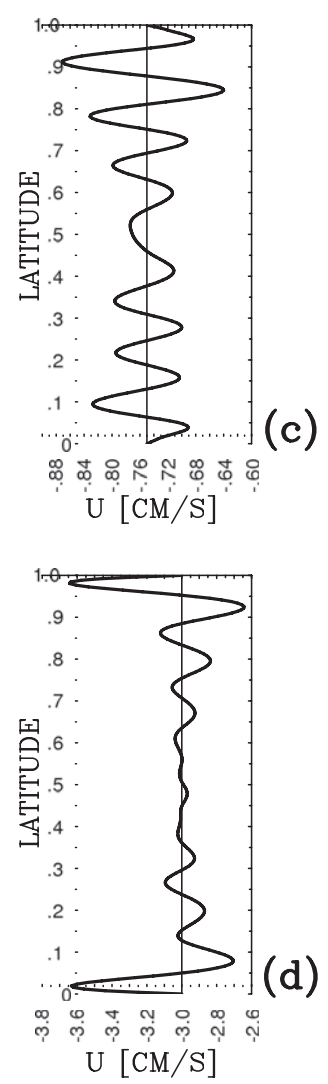

FIG. 15. Multiple-jet flow in the two-layer closed basin. Instantaneous (a) barotropic and (b) baroclinic velocity streamfunctions in the EB flow regime $(\mathrm{CI}=2.5 \mathrm{~Sv})$. (c),(d) The corresponding time-mean zonal velocity profiles show the same quantities as in (a),(b) but for the WB flow regime $(\mathrm{CI}=1 \mathrm{~Sv})$.

We studied the roles of the eddy forcing and its components in maintaining the jets as well as meridional scaling, kinematical properties, and the roles of meridional boundaries. The eddy forcing is a result of the eddy-driven down-gradient mixing of the background PV. It is shown that only in the eastward background flow is the baroclinic component of the jets maintained by the Reynolds stress component of the eddy forcing. This behavior of the Reynolds stresses can be viewed as an example of "negative viscosity" phenomena (Phillips 1956; Starr 1968). In the westward background flow, which has not been studied before, the jets are not only maintained by form stress forcing associated with diverging buoyancy eddy fluxes but they are also are resisted by Reynolds stress forcing. Thus, the eddies maintain the jets by releasing their available potential energy back into the time-mean flow; in this sense, this mechanism is opposite to the baroclinic instability.

We connected our results to several popular theoretical ideas. The Rhines scaling for meridional spacing of the jets is not generally confirmed, and it is also shown that there are multiple equilibria with a different num- ber of the time-mean jets. We find that the Rhines scaling reported in the previous study of $\mathrm{P} 93$ is approximately valid only in a narrow range of parameters.

A theoretical explanation of our findings is presented in Berloff et al. (2009). In particular, the formation, nonlinear dynamics, and equilibration of the jets are explained in terms of linear stability arguments and nonlinear self-interactions of the linear eigenmodes.

We also connected our results to the popular "PV staircase" paradigm (e.g., Baldwin et al. 2007) and found that description of the time-mean meridional PV in terms of the staircase is not accurate. The time-mean flow does not approach the PV staircase paradigm because of the strong barotropic mode predicted by the dynamics. There are alternating weak barriers to and mixers of the meridional material transport that are embedded in the flow. The locations of the barriers and mixers depend on the direction of the background flow and depth, and the barriers are not necessarily located on the prograde jets.

Finally, we find that when the channel is closed with meridional walls, the jet properties are similar to those in 
KBP09: the jets are more latent, more narrow, with the eastward and westward jets of similar strength. However, the meridional walls do not qualitatively change the dynamical balances. Also, in the westward background flow regime, the jets are more intense in the western half of the basin, and they are affected by the weakly damped basin modes. Although these modes modify the flow, they are not the primary mechanism driving the jets. ${ }^{8}$

The following future developments of the results of this paper are anticipated. Spatial inhomogeneity and nonzonality of the background flow (deliberately neglected in this study) might be very important for generating the jets. The intrinsic low-frequency variability of the jets, the roles of the bottom topography, and the dynamical connection between the midlatitude and equatorial zonal jets remain poorly understood.

Acknowledgments. Funding for PB was provided by NSF Grants OCE 0344094 and OCE 0725796 and by the research grant from the Newton Trust of the University of Cambridge. Funding for IK was provided by NSF Grants OCE 0346178 and 0749722 . Funding for JP was provided by NSF Grant OCE 0451086 . PB is also grateful for the stimulating discussions with Peter Haynes, Michael McIntyre, Bill Young, and Andy Thompson.

\section{REFERENCES}

Army, L., 1989: Hydraulic control of zonal currents on a $\beta$-plane. J. Fluid Mech., 201, 357-377.

Baldwin, M., P. Rhines, H.-P. Huang, and M. McIntyre, 2007: The jet-stream conundrum. Science, 315, 467-468.

Balk, A., S. Nazarenko, and V. Zakharov, 1990: On the nonlocal turbulence of drift type waves. Phys. Rev. Lett. A, 146, 217-221.

Berloff, P., 2005: On rectification of randomly forced flows. J. Mar. Res., 63, 497-527.

— J. JcWilliams, and A. Bracco, 2002: Material transport in oceanic gyres. Part I: Phenomenology. J. Phys. Oceanogr., 32, 764-796.

— I. Kamenkovich, and J. Pedlosky, 2009: A mechanism of formation of multiple zonal jets in the oceans. J. Fluid Mech., 628, 395-425.

Beron-Vera, F., M. Brown, M. Olascoaga, I. Rypina, H. Kocak, and I. Udovydchenkov, 2008: Zonal jets as transport barriers in planetary atmospheres. J. Atmos. Sci., 65, 3316-3326.

Chekhlov, A., S. Orszag, S. Sukoriansky, B. Galperin, and I. Staroselsky, 1996: The effect of small-scale forcing on large-scale structures in two-dimensional flows. Physica D., 98, 321-334.

Cox, M., 1987: An eddy-resolving numerical model of the ventilated thermocline: Time dependence. J. Phys. Oceanogr., 17, 1044-1056.

Danilov, S., and V. Gryanik, 2004: Barotropic beta-plane turbulence in a regime with strong zonal jets revisited. J. Atmos. Sci., 61, 2283-2295.

\footnotetext{
${ }^{8}$ The basin-mode mechanism was proposed by Berloff (2005) for the situation without the background shear.
}

— , and D. Gurarie, 2004: Scaling, spectra and zonal jets in betaplane turbulence. Phys. Fluids, 16, 2592-2603.

Diamond, P., S.-I. Itoh, K. Itoh, and T. Hahm, 2005: Zonal flows in plasma-A review. Plasma Phys. Controlled Fusion, 47, R35-R161.

Dritschel, D., and M. McIntyre, 2008: Multiple jets as PV staircases: The Phillips effect and the resilience of eddy-transport barriers. J. Atmos. Sci., 65, 855-874.

Dunkerton, T., and R. Scott, 2008: A barotropic model of the angular momentum-Conserving potential vorticity staircase in spherical geometry. J. Atmos. Sci., 65, 1105-1136.

Esler, G., 2008: The turbulent equilibration of an unstable baroclinic jet. J. Fluid Mech., 599, 241-268.

Farrell, B., and P. Ioannou, 2007: Structure and spacing of jets in barotropic turbulence. J. Atmos. Sci., 64, 3652-3665.

$\longrightarrow$, and - 2008: Formation of jets by baroclinic turbulence. J. Atmos. Sci., 65, 3353-3375.

Firing, E., 1987: Deep zonal currents in the central equatorial Pacific. J. Mar. Res., 45, 791-812.

Franco, B., A. Piola, A. Rivas, A. Baldoni, and J. Pisoni, 2008: Multiple thermal fronts near the Patagonian shelf break. Geophys. Res. Lett., 35, L02607, doi:10.1029/2007GL032066.

Galperin, B., H. Nakano, H.-P. Huang, and S. Sukoriansky, 2004: The ubiquitous zonal jets in the atmospheres of giant planets and Earth's oceans. Geophys. Res. Lett., 31, L13303, doi:10.1029/2004GL019691.

Greenslade, M., and P. Haynes, 2008: Vertical transition in transport and mixing in baroclinic flows. J. Atmos. Sci., 65, 1137-1157.

Haidvogel, D., and I. Held, 1980: Homogeneous quasi-geostrophic turbulence driven by a uniform temperature gradient. J. Atmos. Sci., 37, 2644-2660.

Haynes, P., and E. Shuckburgh, 2000: Effective diffusivity as a diagnostic of atmospheric transport. Part I: Stratosphere. J. Geophys. Res., 105, 22 777-22 794.

- D. Poet, and E. Shuckburgh, 2007: Transport and mixing in kinematic and dynamically consistent flows. J. Atmos. Sci., 64, 3640-3651.

Herbei, R., I. McKeague, and K. Speer, 2008: Gyres and jets: Inversion of tracer data for ocean circulation structure. J. Phys. Oceanogr., 38, 1180-1202.

Hogg, N., and B. Owens, 1999: Direct measurement of the deep circulation within the Brazil basin. Deep-Sea Res. II, 46, 335-353.

Hua, B. L., M. D’Orgeville, M. Fruman, C. Menesguen, R. Schopp, P. Klein, and H. Sasaki, 2008: Destabilization of mixed Rossby-Gravity waves and equatorial zonal jets formation. J. Fluid Mech., 610, 311-341.

Huang, H.-P., and W. Robinson, 1998: Two-dimensional turbulence and persistent zonal jets in a global barotropic model. J. Atmos. Sci., 55, 611-632.

— A. Kaplan, E. Curchitser, and N. Maximenko, 2007: The degree of anisotropy for mid-ocean currents from satellite observations and an eddy-permitting model simulation. J. Geophys. Res., 112, C09005, doi:10.1029/2007JC004105.

Hughes, C., and E. Ash, 2001: Eddy forcing of the mean flow in the Southern Ocean. J. Geophys. Res., 106, 2713-2722.

Ishioka, K., J. Hasegawa, and S. Yoden, 2007: Asymmetrization mechanism of jet profiles in decaying $\beta$-plane turbulence. J. Atmos. Sci., 64, 3340-3353.

Ivanov, L., C. Collins, and T. Margolina, 2009: System of quasizonal jets off California revealed from satellite altimetry. Geophys. Res. Lett., 36, L03609, doi:10.1029/2008GL036327. 
Ivchenko, V., K. Richards, B. Sinha, and J.-O. Wolff, 1997: Parameterization of mesoscale eddy fluxes in zonal ocean flows. J. Mar. Res., 55, 1127-1162.

Juckes, M., and M. McIntyre, 1987: A high resolution, one-layer model of breaking planetary waves in the stratosphere. $\mathrm{Na}$ ture, 328, 590-596.

Kamenkovich, I., P. Berloff, and J. Pedlosky, 2009: Role of eddy forcing in the dynamics of multiple zonal jets in a model of the North Atlantic. J. Phys. Oceanogr., 39, 1361-1379.

Kaspi, I., and G. Flierl, 2007: Formation of jets by baroclinic instability on gas planet atmospheres. J. Atmos. Sci., 64, 3177-3194.

Kondratyev, K., and G. Hunt, 1982: Weather and Climate on Planets. Pergamon Press, $755 \mathrm{pp}$.

Kramer, W., M. van Buren, H. Clercx, and G. van Heijst, 2006: $\beta$-plane turbulence in a basin with no-slip boundaries. Phys. Fluids, 18, 026603, doi:10.1063/1.2173285.

Krauss, W., and C. Boning, 1987: Lagrangian properties of eddy fields in the northern North Atlantic as deduced from satellitetracked buoys. J. Mar. Res., 45, 259-291.

Lapeyre, G., and I. Held, 2003: Diffusivity, kinetic energy dissipation, and closure theories for the poleward eddy heat flux. J. Atmos. Sci., 60, 2907-2916.

Lee, S., 1997: Maintenance of multiple jets in a baroclinic flow. J. Atmos. Sci., 54, 1726-1738.

Manfroi, A., and W. Young, 1999: Slow evolution of zonal jets on the beta plane. J. Atmos. Sci., 56, 784-800.

$\longrightarrow$, and 2002: Stability of $\beta$-plane Kolmogorov flow. Physica D., 162, 208-232.

Marshall, J., E. Shuckburgh, H. Jones, and C. Hill, 2006: Estimates and implications of surface eddy diffusivity in the Southern Ocean derived from tracer transport. J. Phys. Oceanogr., 36, 1806-1821.

Maximenko, N., B. Bang, and H. Sasaki, 2005: Observational evidence of alternating zonal jets in the world ocean. Geophys. Res. Lett., 32, L12607, doi:10.1029/2005GL022728.

_ O. Melnichenko, P. Niiler, and H. Sasaki, 2008: Stationary mesoscale jet-like features in the ocean. Geophys. Res. Lett., 35, L08603, doi:10.1029/2008GL033267.

McIntyre, M., 1982: How well do we understand the dynamics of stratospheric warmings? J. Meteor. Soc. Japan, 60, 37-65.

McWilliams, J., 1977: A note on a consistent quasigeostrophic model in a multiply connected domain. Dyn. Atmos. Oceans, 1, 427-441.

_ 2006: Fundamentals of Geophysical Fluid Dynamics. Cambridge University Press, 249 pp.

Nadiga, B., 2006: On zonal jets in oceans. Geophys. Res. Lett., 33, L10601, doi:10.1029/2006GL025865.

Nakano, H., and H. Hasumi, 2005: A series of zonal jets embedded in the broad zonal flows in the Pacific obtained in eddy-permitting ocean general circulation models. J. Phys. Oceanogr., 35, 474488.

Ollitrault, M., M. Lankhorst, D. Fratantoni, P. Richardson, and W. Zenk, 2006: Zonal intermediate currents in the equatorial Atlantic Ocean. Geophys. Res. Lett., 33, L05605, doi:10.1029/ 2005GL025368.

Panetta, L., 1993: Zonal jets in wide baroclinically unstable regions: Persistence and scale selection. J. Atmos. Sci., 50, 2073-2106.

Pedlosky, J., 1987: Geophysical Fluid Dynamics. 2nd ed. SpringerVerlag, $710 \mathrm{pp}$.

Phillips, N., 1956: The general circulation of the atmosphere: A numerical experiment. Quart. J. Roy. Meteor. Soc., 82, 123-164.
Read, P., Y. H. Yamazaki, S. R. Lewis, P. D. Williams, R. Wordsworth, K. Miki-Yamazaki, J. Sommeria, and H. Didelle, 2007: Dynamics of convectively driven banded jets in the laboratory. J. Atmos. Sci., 64, 4031-4052.

Rhines, P., 1975: Waves and turbulence on a beta-plane. J. Fluid Mech., 69, 417-443.

_ 1994: Jets. Chaos, 4, 313-339.

Richards, K., N. Maximenko, F. Bryan, and H. Sasaki, 2006: Zonal jets in the Pacific ocean. Geophys. Res. Lett., 33, L03605, doi:10.1029/2005GL024645.

Schlax, M., and D. Chelton, 2008: The influence of mesoscale eddies on the detection of quasi-zonal jets in the ocean. Geophys. Res. Lett., 35, L24602, doi:10.1029/2008GL035998.

Scott, R., and L. Polvani, 2007: Forced-dissipative shallow-water turbulence on the sphere and the atmospheric circulation of the giant planets. J. Atmos. Sci., 64, 3158-3176.

Shuckburgh, E., and P. Haynes, 2003: Diagnosing transport and mixing using a tracer-based coordinate system. Phys. Fluids, 15, 3342-3357.

Sinha, B., and K. Richards, 1999: Jet structure and scaling in Southern Ocean models. J. Phys. Oceanogr., 29, 1143-1155.

Smith, K., 2004: A local model for planetary atmospheres forced by small-scale convection. J. Atmos. Sci., 61, 1420-1433.

, and G. Vallis, 2002: The scales and equilibration of midocean eddies: Forced-dissipative flow. J. Phys. Oceanogr., 32, 1699-1720.

- G. Boccaletti, C. Henning, I. Marinov, C. Tam, I. Held, and G. Vallis, 2002: Turbulent diffusion in the geostrophic inverse cascade. J. Fluid Mech., 469, 13-48.

Sokolov, S., and S. Rintoul, 2007a: Multiple jets of the Antarctic Circumpolar Current south of Australia. J. Phys. Oceanogr., 37, 1394-1412.

— , and — 2007b: On the relationship between fronts of the Antarctic Circumpolar Current and surface chlorophyll concentrations in the Southern Ocean. J. Geophys. Res., 112, C07030, doi:10.1029/2006JC004072.

Sommeria, J., S. Meyers, and H. Swinney, 1989: Laboratory model of a planetary eastward jet. Nature, 337, 58-61.

Starr, V., 1968: Physics of Negative Viscosity Phenomena. McGrawHill, 256 pp.

Sukoriansky, S., N. Dikovskaya, and B. Galperin, 2007: On the "arrest" of inverse energy cascade and the Rhines scale. J. Atmos. Sci., 64, 3312-3327.

Theiss, J., 2004: Equatorward energy cascade, critical latitude, and the predominance of cyclonic vortices in geostrophic turbulence. J. Phys. Oceanogr., 34, 1663-1678.

Thompson, A., and W. Young, 2007: Baroclinic eddy heat fluxes: Zonal flows and energy balance. J. Atmos. Sci., 64, 3214-3231.

Treguier, A., and L. Panetta, 1994: Multiple zonal jets in a quasigeostrophic model of the Antarctic Circumpolar Current. J. Phys. Oceanogr., 24, 2263-2277.

- N. Hogg, M. Maltrud, K. Speer, and V. Thierry, 2003: The origin of deep zonal flows in the Brazil basin. J. Phys. Oceanogr., 33, 580-599.

Vallis, G., and M. Maltrud, 1993: Generation of mean flows and jets on a beta plane and over topography. J. Phys. Oceanogr., 23, 1346-1362.

Williams, G., 1978: Planetary circulations: 1. Barotropic representation of Jovian and terrestrial turbulence. J. Atmos. Sci., 35, 1399-1426.

Wordsworth, R., P. Read, and Y. Yamazaki, 2008: Turbulence, waves, and jets in a differentially heated rotating annulus experiment. Phys. Fluids, 20, 126602, doi:10.1063/1.2990042. 